\title{
HOLOMORPHY TYPES AND THE FOURIER-BOREL TRANSFORM BETWEEN SPACES OF ENTIRE FUNCTIONS OF A GIVEN TYPE AND ORDER DEFINED ON BANACH SPACES
}

\author{
VINÍCIUS V. FÁVARO* and ARIOSVALDO M. JATOBÁ
}

\begin{abstract}
Let $E$ be a Banach space and $\Theta$ be a $\pi_{1}$-holomorphy type. The main purpose of this paper is to show that the Fourier-Borel transform is an algebraic isomorphism between the dual of the space $\operatorname{Exp}_{\Theta, A}^{k}(E)$ of entire functions on $E$ of order $k$ and $\Theta$-type strictly less than $A$ and the space $\operatorname{Exp}_{\Theta^{\prime}, 0,(\lambda(k) A)^{-1}}^{k^{\prime}}\left(E^{\prime}\right)$ of entire functions on $E^{\prime}$ of order $k^{\prime}$ and $\Theta^{\prime}$-type less than or equal to $(\lambda(k) A)^{-1}$. The same is proved for the dual of the space $\operatorname{Exp}_{\Theta, A}^{k}(E)$ of entire functions on $E$ of order $k$ and $\Theta$-type less than or equal to $A$ and the space $\operatorname{Exp}_{\Theta^{\prime},(\lambda(k) A)^{-1}}^{k^{\prime}}\left(E^{\prime}\right)$ of entire functions on $E^{\prime}$ of order $k^{\prime}$ and $\Theta^{\prime}$-type strictly less than $(\lambda(k) A)^{-1}$. Moreover, the Fourier-Borel transform is proved to be a topological isomorphism in certain cases.
\end{abstract}

\section{Introduction}

Let $E$ be a Banach space whose dual $E^{\prime}$ has the bounded approximation property. Gupta [6] proved that the Borel transform establishes an algebraic isomorphism between the dual of the space $\mathscr{H}_{\mathrm{Nb}}(E)$ of nuclearly-entire functions of bounded type defined on $E$ and the space $\operatorname{Exp}\left(E^{\prime}\right)$ of entire functions of exponential-type defined on $E^{\prime}$. Matos [11] proved the same for the dual of the space $\mathscr{H}_{\widetilde{\mathrm{N}} \mathrm{b},(s ;(r, q))}(E)$ of $(s ;(r, q))$-quasi-nuclear entire functions of bounded

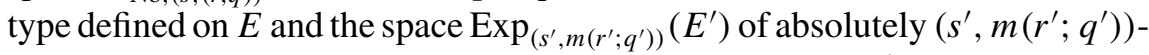
summing entire functions of exponential type defined on $E^{\prime}$. Still in this line, Mujica [15] proved that the Borel transform establishes an algebraic isomorphism between the dual of the space $\mathscr{H}_{\sigma(p) b}(E)$ of $\sigma(p)$-nuclear entire functions of bounded type defined on $E$ and the space $\operatorname{Exp}_{\tau(p)}\left(E^{\prime}\right)$ of absolutely $\tau(p)$ summing entire functions of exponential type defined on $E^{\prime}$. The concept of $\pi_{1}$-holomorphy type is introduced in [3] in order to generalize all these results. There it is proved that if $\Theta$ is a $\pi_{1}$-holomorphy type, then the Borel transform

\footnotetext{
* Supported by Fapemig Grant CEX-APQ-00208-09.

Received 22 July 2010.
} 
establishes an algebraic isomorphism between the dual of the space $\mathscr{H}_{\Theta b}(E)$ of $\Theta$-holomorphy type entire functions of bounded type defined on $E$ and the space $\operatorname{Exp}_{\Theta^{\prime}}\left(E^{\prime}\right)$ of entire functions of $\Theta^{\prime}$-exponential type defined on $E^{\prime}$. The aforementioned results generalize results of this type obtained by Malgrange [7].

A major step in the generalization of the spaces of entire functions of nuclear bounded type defined on $E$ was accomplished in 1984 by Matos [9] through the introduction of the so-called "spaces of nuclear entire functions of a given type and order defined on $E$ ". Such spaces also generalize the spaces of entire functions of a given type and order defined on $C^{n}$ of Martineau [8]. Matos [9] also studied duality results via the Fourier-Borel transform on the dual of these spaces. Using the results obtained in [9], he considered in [10] existence and approximation results for convolution equations defined on spaces of nuclear entire functions of a given type and order.

Following this line of thought, the first author extended the scope of this investigation by considering in [1], [2] the aforementioned results for spaces of entire functions of a given type and order defined using classes of $(s ;(r, q))$ quasi-nuclear polynomials.

The main goal of this paper is to replace these classes of homogeneous polynomials by holomorphy types and $\pi_{1}$-holomorphy types to introduce the spaces of $\Theta$ entire functions of a given type and order defined on $E$ and to prove duality results via the Fourier-Borel transform for these spaces. More precisely, for a given $\pi_{1}$-holomorphy type $\Theta$, in Theorems 4.6 and 4.9 we prove that the Fourier-Borel transform identifies algebraically the dual of $\operatorname{Exp}_{\Theta, 0, A}^{k}(E)$ with $\operatorname{Exp}_{\Theta^{\prime},(\lambda(k) A)^{-1}}^{k^{\prime}}\left(E^{\prime}\right)$. Furthermore in Theorem 4.10 we prove that the Fourier-Borel transform is a topological isomorphism between the dual of $\operatorname{Exp}_{\Theta, A}^{k}(E)$ and $\operatorname{Exp}_{\Theta^{\prime}, 0,(\lambda(k) A)^{-1}}^{k^{\prime}}\left(E^{\prime}\right)$, considering the strong topology on the dual of $\operatorname{Exp}_{\Theta, A}^{k}(E)$. To prove this topological isomorphism we need to characterize the bounded subsets of $\operatorname{Exp}_{\Theta, A}^{k}(E)$.

The paper is organized as follows. Section 2 provides the precise definitions of the spaces we shall work with and the corresponding notation. Some basic results are also proved. In Section 3 we prove the aforementioned characterization of the bounded sets of $\operatorname{Exp}_{\Theta, A}^{k}(E)$. The main results are proved in Section 4. The results we prove can be regarded as a positive answer to a question posed in [3, p. 926].

\section{Notation and Preliminaries Results}

In this work, $\mathrm{N}$ denotes the set of positive integers and $\mathrm{N}_{0}$ denotes the set $\mathrm{N} \cup\{0\}$. The letters $E$ and $F$ will always denote complex Banach spaces and $E^{\prime}$ represents the topological dual of $E$. For $A>0, B_{A}(0)$ denotes the ball 
of center 0 and radius $A$ in a normed space. The space of all continuous $j$ homogeneous polynomials from $E$ into $F$ is denoted by $\mathscr{P}\left({ }^{j} E ; F\right)$ and the space of all holomorphic mappings from an open subset $U \subset E$ into $\mathrm{C}$ is denoted by $\mathscr{H}(U)$. When $F=\mathrm{C}$ we write $\mathscr{P}\left({ }^{j} E\right)$ instead of $\mathscr{P}\left({ }^{j} E ; \mathrm{C}\right)$.

We begin remembering the concept of holomorphy type, introduced by Nachbin [13].

Definition 2.1. A holomorphy type $\Theta$ from $E$ to $F$ is a sequence of Banach spaces $\left(P_{\Theta}\left({ }^{j} E ; F\right)\right)_{j=0}^{\infty}$, the norm on each of them being denoted by $\|\cdot\|_{\Theta}$, such that the following conditions hold true:

(1) Each $P_{\Theta}\left({ }^{j} E ; F\right)$ is a vector subspace of $P\left({ }^{j} E ; F\right)$.

(2) $P_{\Theta}\left({ }^{0} E ; F\right)$ coincides with $P\left({ }^{0} E ; F\right)=F$ as a normed vector space.

(3) There is a real number $\sigma \geq 1$ for which the following is true: given any $k \in \mathrm{N}_{0}, j \in \mathrm{N}_{0}, k \leq j, a \in E$, and $P \in \mathscr{P}_{\Theta}\left({ }^{j} E ; F\right)$, we have

$$
\left.\hat{d}^{k} P(a) \in \mathscr{P}_{\Theta}{ }^{k} E ; F\right), \quad\left\|\frac{1}{k !} \hat{d}^{k} P(a)\right\|_{\Theta} \leq \sigma^{j}\|P\|_{\Theta}\|a\|^{j-k} .
$$

It is obvious that each inclusion $\mathscr{P}_{\Theta}\left({ }^{j} E ; F\right) \subset \mathscr{P}\left({ }^{j} E ; F\right)$ is continuous and $\|P\| \leq \sigma^{j}\|P\|_{\Theta}$.

Definition 2.2. Let $\left(\mathscr{P}_{\Theta}\left({ }^{j} E\right)\right)_{j=0}^{\infty}$ be a holomorphy type from $E$ to C. If $\rho>0$ and $k \geq 1$, we denote by $\mathscr{B}_{\Theta, \rho}^{k}(E)$ the complex vector space of all $f \in \mathscr{H}(E)$ such that $\left.\hat{d}^{j} f(0) \in \mathscr{P}_{\Theta}{ }^{j} E\right)$, for all $j \in \mathrm{N}_{0}$ and

$$
\|f\|_{\Theta, k, \rho}=\sum_{j=0}^{\infty} \rho^{-j}\left(\frac{j}{k e}\right)^{\frac{j}{k}}\left\|\frac{1}{j !} \hat{d}^{j} f(0)\right\|_{\Theta}<+\infty,
$$

normed by $\|\cdot\|_{\Theta, k, \rho}$.

Proposition 2.3. For each $\rho>0$ and $k \geq 1, \mathscr{B}_{\Theta, \rho}^{k}(E)$ is a Banach space.

Proof. Let $\left(f_{n}\right)_{n=1}^{\infty}$ be a Cauchy sequence in $\mathscr{B}_{\Theta, \rho}^{k}(E)$. Given $\varepsilon>0$, there exists $n_{\varepsilon} \in \mathrm{N}$ such that

$$
\sum_{j=0}^{\infty} \rho^{-j}\left(\frac{j}{k e}\right)^{\frac{j}{k}}\left\|\frac{1}{j !}\left(\hat{d}^{j} f_{m}(0)-\hat{d}^{j} f_{n}(0)\right)\right\|_{\Theta}<\varepsilon,
$$

for $m, n \geq n_{\varepsilon}$. This implies that $\left(\hat{d}^{j} f_{n}(0)\right)_{n=1}^{\infty}$ is a Cauchy sequence in $\mathscr{P}_{\Theta}\left({ }^{j} E\right)$, for all $j \in \mathrm{N}_{0}$. Hence there is $P_{j} \in \mathscr{P}_{\Theta}\left({ }^{j} E\right), j \in \mathrm{N}_{0}$, such that

$$
\lim _{n \rightarrow \infty} \hat{d}^{j} f_{n}(0)=P_{j} \text {. }
$$


Thus making $n \rightarrow \infty$ in (1), we have

$$
\sum_{j=0}^{\infty} \rho^{-j}\left(\frac{j}{k e}\right)^{\frac{j}{k}}\left\|\frac{1}{j !}\left(\hat{d}^{j} f_{m}(0)-P_{j}\right)\right\|_{\Theta}<\varepsilon,
$$

for all $m \geq n_{\varepsilon}$. Set

$$
f(x)=\sum_{j=0}^{\infty} \frac{1}{j !} P_{j}(x),
$$

for all $x \in E$. To prove that $f \in \mathscr{H}(E)$, it is enough to show that

$$
\limsup _{j \rightarrow \infty}\left\|\frac{1}{j !} P_{j}\right\|^{\frac{1}{j}}=0 .
$$

Note that,

$$
\begin{aligned}
\rho^{-j}\left(\frac{j}{k e}\right)^{\frac{j}{k}}\left\|\frac{1}{j !} P_{j}\right\|_{\Theta} & \leq \rho^{-j}\left(\frac{j}{k e}\right)^{\frac{j}{k}}\left\|\frac{1}{j !}\left(\hat{d}^{j} f_{n_{\varepsilon}}(0)-P_{j}\right)\right\|_{\Theta} \\
& +\rho^{-j}\left(\frac{j}{k e}\right)^{\frac{j}{k}}\left\|\frac{1}{j !} \hat{d}^{j} f_{n_{\varepsilon}}(0)\right\|_{\Theta} \\
\leq & \varepsilon+\left\|f_{n_{\varepsilon}}\right\|_{\Theta, k, \rho} .
\end{aligned}
$$

This implies that

$$
\limsup _{j \rightarrow \infty}\left\|\frac{1}{j !} P_{j}\right\|_{\Theta}^{\frac{1}{j}} \leq \limsup _{j \rightarrow \infty} \frac{\rho(k e)^{\frac{1}{k}}\left(\varepsilon+\left\|f_{n_{\varepsilon}}\right\|_{\Theta, k, \rho}\right)^{\frac{1}{j}}}{j^{\frac{1}{k}}}=0 .
$$

Since $\left\|P_{j}\right\| \leq \sigma^{j}\left\|P_{j}\right\|_{\Theta}$, we have

$$
\limsup _{j \rightarrow \infty}\left\|\frac{1}{j !} P_{j}\right\|^{\frac{1}{j}} \leq \sigma \limsup _{j \rightarrow \infty}\left\|\frac{1}{j !} P_{j}\right\|_{\Theta}^{\frac{1}{j}}=0 .
$$

Now

$$
\|f\|_{\Theta, k, \rho} \leq\left\|f-f_{n_{\varepsilon}}\right\|_{\Theta, k, \rho}+\left\|f_{n_{\varepsilon}}\right\|_{\Theta, k, \rho}<\varepsilon+\left\|f_{n_{\varepsilon}}\right\|_{\Theta, k, \rho}<+\infty,
$$

Hence $f \in \mathscr{B}_{\Theta, \rho}^{k}(E)$ and by (2) we conclude that $\left(f_{n}\right)_{n=1}^{\infty}$ converges to $f$.

Definition 2.4. Let $\left(\mathscr{P}_{\Theta}\left({ }^{j} E\right)\right)_{j=0}^{\infty}$ be a holomorphy type from $E$ to $\mathrm{C}$. If $A \in(0,+\infty)$ and $k \geq 1$, we denote by $\operatorname{Exp}_{\Theta, A}^{k}(E)$ the complex vector space $\cup_{\rho<A} \mathscr{B}_{\Theta, \rho}^{k}(E)$ with the locally convex inductive limit topology. We 
consider the complex vector space $\operatorname{Exp}_{\Theta, 0, A}^{k}(E)=\bigcap_{\rho>A} \mathscr{B}_{\Theta, \rho}^{k}(E)$ with the projective limit topology. If $A=+\infty$ and $k \geq 1$, we consider the complex vector space $\operatorname{Exp}_{\Theta, \infty}^{k}(E)=\bigcup_{\rho>0} \mathscr{B}_{\Theta, \rho}^{k}(E)$ with the locally convex inductive limit topology and if $A=0$ and $k \geq 1$, we consider the complex vector spaces $\operatorname{Exp}_{\Theta, 0}^{k}(E)=\operatorname{Exp}_{\Theta, 0,0}^{k}(E)=\bigcap_{\rho>0} \mathscr{B}_{\Theta, \rho}^{k}(E)$ with the locally convex projective limit topology.

As simple consequences of Definition 2.4 we have the following characterizations:

Proposition 2.5. Let $\left(\mathscr{P}_{\Theta}\left({ }^{j} E\right)\right)_{j=0}^{\infty}$ be a holomorphy type from $E$ to $\mathrm{C}$ and $k \in[1,+\infty)$. If $f \in \mathscr{H}(E)$ is such that $\hat{d}^{j} f(0) \in \mathscr{P}_{\Theta}\left({ }^{j} E\right), \forall j \in \mathrm{N}_{0}$, then

(a) For each $A \in(0,+\infty], f \in \operatorname{Exp}_{\Theta, A}^{k}(E)$ if, and only if,

$$
\limsup _{j \rightarrow \infty}\left(\frac{j}{k e}\right)^{\frac{1}{k}}\left\|\frac{1}{j !} \hat{d}^{j} f(0)\right\|_{\Theta}^{\frac{1}{j}}<A .
$$

(b) For each $A \in[0,+\infty), f \in \operatorname{Exp}_{\Theta, 0, A}^{k}(E)$ if, and only if,

$$
\limsup _{j \rightarrow \infty}\left(\frac{j}{k e}\right)^{\frac{1}{k}}\left\|\frac{1}{j !} \hat{d}^{j} f(0)\right\|_{\Theta}^{\frac{1}{j}} \leq A .
$$

Proof. (a) If $f \in \operatorname{Exp}_{\Theta, A}^{k}(E)$, then there exists $\rho<A$ such that $f \in$ $\mathscr{B}_{\Theta, \rho}^{k}(E)$ and

$$
\sum_{j=0}^{\infty} \rho^{-j}\left(\frac{j}{k e}\right)^{\frac{j}{k}}\left\|\frac{1}{j !} \hat{d}^{j} f(0)\right\|_{\Theta}<+\infty .
$$

Thus

$$
\frac{1}{r_{c}}=\limsup _{j \rightarrow \infty}\left(\frac{j}{k e}\right)^{\frac{1}{k}}\left\|\frac{1}{j !} \hat{d}^{j} f(0)\right\|_{\Theta}^{\frac{1}{j}} \leq \rho<A,
$$

where $r_{c}$ denotes the radius of convergence of the power series

$$
\sum_{j=0}^{\infty}\left(\frac{j}{k e}\right)^{\frac{j}{k}}\left\|\frac{1}{j !} \widehat{d}^{j} f(0)\right\|_{\Theta} z^{j}, \quad z \in \mathrm{C} .
$$

On the other hand, if

$$
\alpha=\limsup _{j \rightarrow \infty}\left(\frac{j}{k e}\right)^{\frac{1}{k}}\left\|\frac{1}{j !} \hat{d}^{j} f(0)\right\|_{\Theta}^{\frac{1}{j}}<A,
$$


then, for all $\rho \in] \alpha, A[$, the series

$$
\sum_{j=0}^{\infty} \rho^{-j}\left(\frac{j}{k e}\right)^{\frac{j}{k}}\left\|\frac{1}{j !} \hat{d}^{j} f(0)\right\|_{\Theta}
$$

is convergent. Thus $f \in \mathscr{B}_{\Theta, \rho}^{k}(E)$ and so $f \in \operatorname{Exp}_{\Theta, A}^{k}(E)$. Note that this proof applies to $A=+\infty$.

(b) If $f \in \operatorname{Exp}_{\Theta, 0, A}^{k}(E)$, then $f \in \mathscr{B}_{\Theta, \rho}^{k}(E)$, for all $\rho>A$. Thus,

$$
\limsup _{j \rightarrow \infty}\left(\frac{j}{k e}\right)^{\frac{1}{k}}\left\|\frac{1}{j !} \hat{d}^{j} f(0)\right\|_{\Theta}^{\frac{1}{j}} \leq \rho,
$$

for all $\rho>A$ and consequently

$$
\limsup _{j \rightarrow \infty}\left(\frac{j}{k e}\right)^{\frac{1}{k}}\left\|\frac{1}{j !} \hat{d}^{j} f(0)\right\|_{\Theta}^{\frac{1}{j}} \leq A .
$$

Conversely, if

$$
\limsup _{j \rightarrow \infty}\left(\frac{j}{k e}\right)^{\frac{1}{k}}\left\|\frac{1}{j !} \hat{d}^{j} f(0)\right\|_{\Theta}^{\frac{1}{j}} \leq A,
$$

it follows that

$$
\limsup _{j \rightarrow \infty}\left(\frac{j}{k e}\right)^{\frac{1}{k}}\left\|\frac{1}{j !} \hat{d}^{j} f(0)\right\|_{\Theta}^{\frac{1}{j}}<\rho,
$$

for all $\rho>A$. Thus, $f \in \mathscr{B}_{\Theta, \rho}^{k}(E)$, for all $\rho>A$ and so $f \in \operatorname{Exp}_{\Theta, 0, A}^{k}(E)$.

Note that this proof applies to $A=0$.

Remark 2.6. Let us recall the original definition, due to Nachbin [12, p. 226]: a function $f \in \mathscr{H}(E)$ is said to be of exponential type strictly less than $A$ if, for each $\varepsilon>0$, there is $c>0$ such that $|f(x)| \leq c e^{(A+\varepsilon)\|x\|}$ for every $x \in E$. He also proved that this occurs if and only if $\lim _{\lim } \sup _{j \rightarrow \infty}\left\|\hat{d}^{j} f(0)\right\|^{\frac{1}{j}}<$ $A$. In the same fashion, under the hypothesis of Proposition 2.5 we have the following characterizations:

(a) $f \in \operatorname{Exp}_{\Theta, A}^{1}(E)$ if, and only if, $\limsup _{j \rightarrow \infty}\left\|\hat{d}^{j} f(0)\right\|_{\Theta}^{\frac{1}{j}}<A$.

(b) $f \in \operatorname{Exp}_{\Theta, 0, A}^{1}(E)$ if, and only if, $\limsup _{j \rightarrow \infty}\left\|\hat{d}^{j} f(0)\right\|_{\Theta}^{\frac{1}{j}} \leq A$.

To prove them, it is enough to use that $\lim _{j \rightarrow \infty} \frac{j}{e}\left(\frac{1}{j !}\right)^{\frac{1}{j}}=1$. 
Due to these results and to the original terminology of Nachbin, the elements of $\operatorname{Exp}_{\Theta, A}^{k}(E)$ are called entire functions of order $k$ and $\Theta$-exponential type strictly less than $A$. For $A=+\infty$ we drop out "strictly less than $A$ ".

We call the elements of $\operatorname{Exp}_{\Theta, 0, A}^{k}(E)$ entire functions of order $k$ and $\Theta$ exponential type less than or equal to $A$.

The next result shows that the spaces of Definition 2.4 have special structures.

Proposition 2.7. Let $\left(\mathscr{P}_{\Theta}\left({ }^{j} E\right)\right)_{j=0}^{\infty}$ be a holomorphy type from $E$ to $\mathrm{C}$.

(a) For each $A \in(0,+\infty]$ and $k>1, \operatorname{Exp}_{\Theta, A}^{k}(E)$ is a $D F$-space.

(b) For each $A \in[0,+\infty)$ and $k>1, \operatorname{Exp}_{\Theta, 0, A}^{k}(E)$ is a Fréchet space.

Proof. Let $\left(a_{n}\right)_{n=1}^{\infty}$ be a strictly increasing sequence of positive real numbers, converging to $A$. Hence $\bigcup_{\rho<A} \mathscr{B}_{\Theta, \rho}^{k}(E)=\bigcup_{n=1}^{\infty} \mathscr{B}_{\Theta, a_{n}}^{k}(E)$ and it is not difficult to prove that the inductive limit topologies given by $\mathscr{B}_{\Theta, \rho}^{k}(E), \rho<A$ and $\mathscr{B}_{\Theta, a_{n}}^{k}(E), n \in \mathrm{N}$, are equal. Since the inductive limit of a sequence of $D F$-spaces is a $D F$-space (see Grothendieck [5, p. 171, Proposition 5]) we have that $\operatorname{Exp}_{\Theta, A}^{k}(E)$ is a $D F$-space and (a) is proved.

Now we prove (b). We have that $\operatorname{Exp}_{\Theta, 0, A}^{k}(E)$ is a complete locally convex space, since it is the projective limit of complete Hausdorff locally convex spaces (see [14, Proposition V.11]). Let $\left(b_{n}\right)_{n=1}^{\infty}$ be a strictly decreasing sequence of positive real numbers, converging to $A$. Since the topology of the $\operatorname{Exp}_{\Theta, 0, A}^{k}(E)$ and the topology generated by $\|\cdot\|_{\Theta, b_{n}}, n \in \mathrm{N}$, coincide, then $\operatorname{Exp}_{\Theta, 0, A}^{k}(E)$ is metrizable.

In order to consider similar spaces of functions of infinite order, we introduce some new definitions.

Definition 2.8. Let $\left(\mathscr{P}_{\Theta}\left({ }^{j} E\right)\right)_{j=0}^{\infty}$ be a holomorphy type from $E$ to C. If $A \in[0,+\infty)$, we denote by $\mathscr{H}_{\Theta b}\left(B_{\frac{1}{A}}(0)\right)$ the complex vector space of all $f \in \mathscr{H}\left(B_{\frac{1}{A}}(0)\right)$ such that $\hat{d}^{j} f(0) \in \mathscr{P}_{\Theta}\left({ }^{j} E\right)$, for all $j \in \mathrm{N}_{0}$ and

$$
\underset{j \rightarrow \infty}{\limsup }\left\|\frac{1}{j !} \hat{d}^{j} f(0)\right\|_{\Theta}^{\frac{1}{j}} \leq A,
$$

endowed with the locally convex topology generated by the family of seminorms $\left(p_{\Theta, \rho}^{\infty}\right)_{\rho>A}$, where

$$
p_{\Theta, \rho}^{\infty}(f)=\sum_{j=0}^{\infty} \rho^{-j}\left\|\frac{1}{j !} \hat{d}^{j} f(0)\right\|_{\Theta} .
$$


We also denote $\mathscr{H}_{\Theta b}\left(B_{\frac{1}{A}}(0)\right)$ by $\operatorname{Exp}_{\Theta, 0, A}^{\infty}(E)$ and we also write $\operatorname{Exp}_{\Theta, 0}^{\infty}(E)$ $=\operatorname{Exp}_{\Theta, 0,0}^{\infty}(E)$. Note that the space $\operatorname{Exp}_{\Theta, 0}^{\infty}(E)$ coincides with the space $\mathscr{H}_{\Theta b}(E)$ of [3].

Proposition 2.9. Let $\left(\mathscr{P}_{\Theta}\left({ }^{j} E\right)\right)_{j=0}^{\infty}$ be a holomorphy type from $E$ to $\mathrm{C}$. If $A \in[0,+\infty)$, then $\mathscr{H}_{\Theta b}\left(B_{\frac{1}{A}}(0)\right)$ is a Fréchet space.

Proof. Let $\left(a_{n}\right)_{n=1}^{\infty}$ be a strictly decreasing sequence of positive real numbers, converging to $A$. Since $\left(p_{\Theta, a_{n}}^{\infty}\right)_{n=1}^{\infty}$ and $\left(p_{\Theta, \rho}^{\infty}\right)_{\rho>A}$ generate the same topology, we have that $\mathscr{H}_{\Theta b}\left(B_{\frac{1}{A}}(0)\right)$ is a locally convex and metrizable topological vector space. Let $\left(f_{k}\right)_{k=1}^{\infty}$ be a Cauchy sequence in $\mathscr{H}_{\Theta b}\left(B_{\frac{1}{A}}(0)\right)$ and $\rho>A$. Proceeding as in Proposition 2.3 we conclude that

$$
p_{\Theta, \rho}^{\infty}\left(f_{k}-f\right) \rightarrow 0, \quad \text { when } \quad k \rightarrow \infty .
$$

Hence $\mathscr{H}_{\Theta b}\left(B_{\frac{1}{A}}(0)\right)$ is complete.

We next define certain spaces of germs of holomorphic functions.

Let $L=\bigcup_{\rho<A} \mathscr{H}_{\Theta}\left(B_{\frac{1}{\rho}}(0)\right)$ and define the following equivalence relation:

$$
f \sim g \Longleftrightarrow \text { there is } \rho \in(0, A) \text { such that }\left.f\right|_{B_{\frac{1}{\rho}}(0)}=\left.g\right|_{B_{\frac{1}{\rho}}(0)} .
$$

We denote by $L / \sim$ the set of all equivalence classes of elements of $L$ and by $[f]$ the equivalence class which has $f$ as one representative. If we define the operations

$$
[f]+[g]=\left[\left.f\right|_{B_{\frac{1}{\rho}}(0)}+\left.g\right|_{B_{\frac{1}{\rho}}(0)}\right],
$$

where $\rho \in(0, A)$ is such that $\left.f\right|_{B_{\frac{1}{\rho}}(0)},\left.g\right|_{B_{\frac{1}{\rho}}(0)} \in \mathscr{H}_{\Theta b}\left(B_{\frac{1}{\rho}}(0)\right)$, and

$$
\lambda[f]=[\lambda f], \quad \lambda \in \mathrm{C},
$$

then $L / \sim$ becomes a vector space. For each $\rho \in(0, A)$, let $i_{\rho}: \mathscr{H}_{\Theta b}\left(B_{\frac{1}{\rho}}(0)\right) \rightarrow$ $L / \sim$ be given by $i_{\rho}(f)=[f]$.

Definition 2.10. For $A \in(0,+\infty]$, we define $\mathscr{H}_{\Theta b}\left(\overline{B_{\frac{1}{A}}(0)}\right)=L / \sim$ with the locally convex inductive limit topology generated by the family $\left(i_{\rho}\right)_{\rho \in(0, A)}$.

An equivalent way to introduce this space is the following:

Definition 2.11. Let $\left(\mathscr{P}_{\Theta}\left({ }^{j} E\right)\right)_{j=0}^{\infty}$ be a holomorphy type from $E$ to C. For $\rho>0$, we define the complex vector space $\mathscr{H}_{\Theta}^{\infty}\left(B_{\frac{1}{\rho}}(0)\right)$ of all $f \in \mathscr{H}_{\left(B_{\frac{1}{\rho}}(0)\right)}$ such that $\left.\hat{d}^{j} f(0) \in \mathscr{P}_{\Theta}{ }^{j} E\right)$, for all $j \in \mathrm{N}_{0}$ and

$$
\sum_{j=0}^{\infty} \rho^{-j}\left\|\frac{1}{j !} \hat{d}^{j} f(0)\right\|_{\Theta}<+\infty,
$$


which is a Banach space with the norm $p_{\Theta, \rho}^{\infty}$ introduced in Definition 2.8.

As in Definition 2.10, we consider an equivalence relation on $L=$ $\bigcup_{\rho<A} \mathscr{H}_{\Theta}^{\infty}\left(B_{\frac{1}{\rho}}(0)\right)$, and for $A \in(0,+\infty]$, we define $\operatorname{Exp}_{\Theta, A}^{\infty}(E)=L / \sim=$ $\bigcup_{\rho<A} \mathscr{H}_{\Theta}^{\infty}\left(B_{\frac{1}{\rho}}(0)\right) / \sim$ with the locally convex inductive limit topology.

It is not difficult to prove that $\mathscr{H}_{\Theta b}\left(\overline{B_{\frac{1}{A}}(0)}\right)$ and $\operatorname{Exp}_{\Theta, A}^{\infty}(E)$ coincide algebraically and are topologically isomorphic.

Due to this definition we denote the space $\mathscr{H}_{\Theta b}\left(\overline{B_{\frac{1}{A}}(0)}\right)$ by $\operatorname{Exp}_{\Theta, A}^{\infty}(E)$, and since $\lim _{k \rightarrow \infty}\left(\frac{j}{k e}\right)^{\frac{1}{k}}=1$, we use the notation $\|\cdot\|_{\Theta, \infty, \rho}$ instead of $p_{\Theta, \rho}^{\infty}$.

Proposition 2.12. Let $\left(\mathscr{P}_{\Theta}\left({ }^{j} E\right)\right)_{j=0}^{\infty}$ be a holomorphy type from $E$ to $\mathrm{C}$. Then

(a) For each $A \in(0,+\infty], \operatorname{Exp}_{\Theta, A}^{\infty}(E)$ is a DF-space.

(b) For each $A \in[0,+\infty)$, $\operatorname{Exp}_{\Theta, 0, A}^{\infty}(E)$ is a Fréchet space.

Proof. Note that (b) is exactly Proposition 2.9 and the proof of (a) follows as in Proposition 2.7.

Proposition 2.13. Let $\left(\mathscr{P}_{\Theta}\left({ }^{j} E\right)\right)_{j=0}^{\infty}$ be a holomorphy type from $E$ to $\mathrm{C}$.

(a) If $k \in[1,+\infty]$ and $A \in(0,+\infty]$, then the Taylor series at 0 for each element of $\operatorname{Exp}_{\Theta, 0, A}^{k}(E)$ converges to the element in the topology of the space.

(b) If $k \in[1,+\infty]$ and $A \in[0,+\infty)$, then the Taylor series at 0 for each element of $\operatorname{Exp}_{\Theta, A}^{k}(E)$ converges to the element in the topology of the space.

PROOF. For each $f$ in the appropriate space we have

$$
\begin{gathered}
\left\|f-\sum_{j=0}^{n} \frac{1}{j !} \hat{d}^{j} f(0)\right\|_{\Theta, k, \rho} \\
=\sum_{j=n+1}^{\infty} \rho^{-j}\left(\frac{j}{k e}\right)^{\frac{j}{k}}\left\|\frac{1}{j !} \hat{d}^{j} f(0)\right\|_{\Theta}, \quad k \in[1,+\infty) . \\
p_{\Theta, \rho}^{\infty}\left(f-\sum_{j=0}^{n} \frac{1}{j !} \hat{d}^{j} f(0)\right)=\sum_{j=n+1}^{\infty} \rho^{-j}\left\|\frac{1}{j !} \hat{d}^{j} f(0)\right\|_{\Theta}
\end{gathered}
$$

Since the topology of $\operatorname{Exp}_{\Theta, 0, A}^{k}(E), k \in[1,+\infty]$, is generated by the corresponding families of seminorms and since (4) and (5) are valid for the respective $\rho>0$, we have the convergence on $\operatorname{Exp}_{\Theta, 0, A}^{k}(E)$ when $n$ tends to infinity. Now

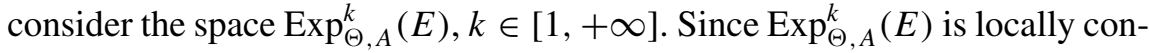
vex, there is a family of seminorms $\left(q_{i}\right)_{i \in I}$, where $I$ is a directed set, that 
defines the topology of it. Since the inclusion $i_{\rho}: \mathscr{B}_{\Theta, \rho}^{k}(E) \rightarrow \operatorname{Exp}_{\Theta, A}^{k}(E)$ is continuous for all $\rho<A$, then there exists $C>0$ such that

$$
q_{i}(g)=q_{i}\left(i_{\rho}(g)\right) \leq C\|g\|_{\Theta, k, \rho},
$$

for all $g \in \mathscr{B}_{\Theta, \rho}^{k}(E)$ and $i \in I$. Let $g=f-\sum_{j=0}^{n} \frac{1}{j !} \hat{d}^{j} f(0)$. Since (4) and (5) go to zero when $n$ tends to infinity, it follows from (6) that $q_{i}\left(\sum_{j=0}^{n} \frac{1}{j !} \hat{d}^{j} f(0)\right)$ $\rightarrow q_{i}(f)$ and the result is proved.

Now we are interested in proving that the exponential function $e^{\varphi}, \varphi \in$ $E^{\prime}$, belongs to the spaces in question. To prove this we need that the holomorphy type $\Theta$ has some properties, more specifically we need that $\Theta$ be a $\pi_{1}$-holomorphy type. This concept was introduced in [3] and we remember it below.

Definition 2.14. Let $\left(\mathscr{P}_{\Theta}\left({ }^{j} E ; F\right)\right)_{j=0}^{\infty}$ be a holomorphy type from $E$ to $F$. The holomorphy type $\Theta$ is said to be a $\pi_{1}$-holomorphy type if the following conditions hold:

(1) $\left\|\phi^{j} \otimes b\right\|_{\Theta}=\|\phi\|^{j}\|b\|$ for all $\phi \in E^{\prime}, b \in F$ and $j \in \mathrm{N}_{0}$;

(2) For each $j \in \mathrm{N}_{0}, \mathscr{P}_{f}\left({ }^{j} E ; F\right)$ is dense in $\left(\mathscr{P}_{\Theta}\left({ }^{j} E ; F\right),\|\cdot\|_{\Theta}\right)$, where $\left.\mathscr{P}_{f}{ }^{j} E ; F\right)$ denotes the space of all $j$-homogeneous polynomials of finite type.

Proposition 2.15. Let $\left(\mathscr{P}_{\Theta}\left({ }^{j} E\right)\right)_{j=0}^{\infty}$ be a $\pi_{1}$-holomorphy type from $E$ to $\mathrm{C}$.

(a) If $k \in(1,+\infty]$ and $A \in(0,+\infty]$, then $e^{\varphi}$ belongs to $\operatorname{Exp}_{\Theta, A}^{k}(E)$, for all $\varphi \in E^{\prime}$.

(b) If $k \in(1,+\infty]$ and $A \in[0,+\infty)$, then $e^{\varphi}$ belongs to $\operatorname{Exp}_{\Theta, 0, A}^{k}(E)$, for all $\varphi \in E^{\prime}$.

(c) If $k=1$ and $A \in(0,+\infty]$, then $e^{\varphi}$ belongs to $\operatorname{Exp}_{\Theta, A}^{1}(E)$, for all $\varphi \in E^{\prime}$ such that $\|\varphi\|<A$.

(d) If $k=1$ and $A \in(0,+\infty]$, then $e^{\varphi}$ belongs to $\operatorname{Exp}_{\Theta, 0, A}^{1}(E)$, for all $\varphi \in E^{\prime}$ such that $\|\varphi\| \leq A$.

Proof. By Definition 2.14 (1) we have $\left\|\hat{d}^{j}\left(e^{\varphi}\right)(0)\right\|_{\Theta}=\|\varphi\|^{j}$. Let $k \in$ $(1,+\infty)$, then $\frac{1-k}{k}<0$ and

$$
\limsup _{j \rightarrow \infty}\left(\frac{j}{e}\right)^{\frac{1-k}{k}}=0 .
$$


Thus

$$
\begin{gathered}
\limsup _{j \rightarrow \infty}\left(\frac{j}{k e}\right)^{\frac{1}{k}}\left(\frac{1}{j !}\right)^{\frac{1}{j}}\left\|\hat{d}^{j}\left(e^{\varphi}\right)(0)\right\|_{\Theta}^{\frac{1}{j}} \\
=\limsup _{j \rightarrow \infty}\left(\frac{j}{k e}\right)^{\frac{1}{k}}\left(\frac{1}{j !}\right)^{\frac{1}{j}}\|\varphi\| \\
=\|\varphi\|\left(\frac{1}{k}\right)^{\frac{1}{k}} \limsup _{j \rightarrow \infty}\left(\frac{j}{e}\right)^{\frac{1-k}{k}}\left(\frac{j}{e}\right)\left(\frac{1}{j !}\right)^{\frac{1}{j}} \\
=\|\varphi\|\left(\frac{1}{k}\right)^{\frac{1}{k}} \limsup _{j \rightarrow \infty}\left(\frac{j}{e}\right)^{\frac{1-k}{k}}=0
\end{gathered}
$$

and it follows from Proposition 2.5 that (a) and (b) are proved, for $k \in(1,+\infty)$. If $k=+\infty$, we have

$$
\limsup _{j \rightarrow \infty}\left\|\frac{1}{j !} \hat{d}^{j}\left(e^{\varphi}\right)(0)\right\|_{\Theta}^{\frac{1}{j}}=\limsup _{j \rightarrow \infty}\left(\frac{1}{j !}\right)^{\frac{1}{j}}\|\varphi\|=0,
$$

Thus (a) follows from Definition 2.8 and (b) follows from Definition 2.11. Now we prove (c) and (d). Since

$$
\limsup _{j \rightarrow \infty} \frac{j}{e}\left(\frac{1}{j !}\right)^{\frac{1}{j}}\left\|\hat{d}^{j}\left(e^{\varphi}\right)(0)\right\|_{\Theta}^{\frac{1}{j}}=\limsup _{j \rightarrow \infty}\|\varphi\|=\|\varphi\|,
$$

then (c) and (d) follow from Proposition 2.5 when $\|\varphi\|<A$ and $\|\varphi\| \leq A$, respectively.

Proposition 2.16. Let $\left(\mathscr{P}_{\Theta}\left({ }^{j} E\right)\right)_{j=0}^{\infty}$ be a $\pi_{1}$-holomorphy type from $E$ to $\mathrm{C}$.

(a) The vector subspace generated by all $e^{\varphi}, \varphi \in E^{\prime}$, is dense in:

(a.1) $\operatorname{Exp}_{\Theta, A}^{k}(E)$ if $k \in(1,+\infty]$ and $A \in(0,+\infty]$.

(a.2) $\operatorname{Exp}_{\Theta, 0, A}^{k}(E)$ if $k \in(1,+\infty]$ and $A \in[0,+\infty)$.

(b) The vector subspace generated by $e^{\varphi}, \varphi \in E^{\prime},\|\varphi\|<A$, is dense in $\operatorname{Exp}_{\Theta, A}^{1}(E)$ if $A \in(0,+\infty)$.

(c) The vector subspace generated by $e^{\varphi}, \varphi \in E^{\prime},\|\varphi\| \leq A$, is dense in $\operatorname{Exp}_{\Theta, 0, A}^{1}(E)$ if $A \in(0,+\infty)$.

Proof. Let $g$ be the closure of $\operatorname{Exp}_{\Theta, A}^{k}(E), k \in(1,+\infty]$. By Definition $2.14(2), \mathscr{P}_{f}\left({ }^{j} E\right)$ is dense in $\mathscr{P}_{\Theta}\left({ }^{j} E\right)$, for all $j \in \mathrm{N}_{0}$. Using this fact in addition with Proposition 2.13 we only have to show that $\mathscr{P}_{f}\left({ }^{j} E\right) \subseteq g$, for each $j \in \mathrm{N}_{0}$. Now the result follows using similar arguments to that used in [1, Proposition 2.16]. The other cases also follow using analogue arguments. 


\section{Bounded Sets}

As mentioned in the Introduction, in order to prove that the Fourier-Borel transform is sometimes a topological isomorphism we need to characterize the bounded subsets of $\operatorname{Exp}_{\Theta, A}^{k}(E), k \in[1,+\infty], A \in(0,+\infty]$. We also characterize the bounded subsets of $\operatorname{Exp}_{\Theta, 0, A}^{k}(E), k \in[1,+\infty], A \in[0,+\infty)$, in order to prove that the inverse of the Fourier-Borel transform defined on the dual of $\operatorname{Exp}_{\Theta, 0, A}^{k}(E)$ is continuous.

We denote by $\mathscr{S}_{A}$ the family of all sequences $\alpha=\left(\alpha_{j}\right)_{j=0}^{\infty}$ of real numbers $\alpha_{j} \geq 0$, such that $\lim \sup _{j \rightarrow \infty} \alpha_{j}^{\frac{1}{j}} \leq A$.

Proposition 3.1. For $k \in[1,+\infty), A \in(0,+\infty]$ and $\alpha \in \mathscr{S}_{\frac{1}{A}}$, the seminorm $p_{\Theta, k, \alpha}$ defined by

$$
p_{\Theta, k, \alpha}(f)=\sum_{j=0}^{\infty} \alpha_{j}\left(\frac{j}{k e}\right)^{\frac{j}{k}}\left\|\frac{\hat{d}^{j} f(0)}{j !}\right\|_{\Theta}
$$

is continuous on $\operatorname{Exp}_{\Theta, A}^{k}(E)$.

For $k=+\infty, A \in(0,+\infty]$ and $\alpha \in \mathscr{I}_{\frac{1}{A}}$ the seminorm $p_{\Theta, \infty, \alpha}$ defined by

$$
p_{\Theta, \infty, \alpha}(f)=\sum_{j=0}^{\infty} \alpha_{j}\left\|\frac{\hat{d}^{j} f(0)}{j !}\right\|_{\Theta}
$$

is continuous on $\operatorname{Exp}_{\Theta, A}^{\infty}(E)$.

Proof. For $\rho \in(0, A)$, we have $\frac{1}{A}<\frac{1}{\rho}$. Since $\alpha \in \mathscr{S}_{\frac{1}{A}}$, there is $C(\rho)>0$ such that $\alpha_{j} \leq C(\rho) \frac{1}{\rho^{j}}$, for all $j \in \mathrm{N}_{0}$, Thus,

$$
p_{\Theta, k, \alpha}(f) \leq C(\rho) \sum_{j=0}^{\infty} \frac{1}{\rho^{j}}\left(\frac{j}{k e}\right)^{\frac{j}{k}}\left\|\frac{\hat{d}^{j} f(0)}{j !}\right\|_{\Theta}=C(\rho)\|f\|_{\Theta, k, \rho},
$$

for all $k \in[1,+\infty)$ and

$$
p_{\Theta, \infty, \alpha}(f) \leq C(\rho) \sum_{j=0}^{\infty} \frac{1}{\rho^{j}}\left\|\frac{\hat{d}^{j} f(0)}{j !}\right\|_{\Theta}=C(\rho) p_{\Theta, \rho}^{\infty}(f) .
$$

Hence $p_{\Theta, k, \alpha}$ and $p_{\Theta, \infty, \alpha}$ are continuous in $\operatorname{Exp}_{\Theta, A}^{k}(E)$ and $\operatorname{Exp}_{\Theta, A}^{\infty}(E)$, respectively. 
Proposition 3.2. For $k \in[1,+\infty)$ and $A \in(0,+\infty]$, a subset $B$ of $\operatorname{Exp}_{\Theta, A}^{k}(E)$ is bounded if, and only if, there is $\rho \in(0, A)$ such that

$$
\limsup _{j \rightarrow \infty}\left(\frac{j}{k e}\right)^{\frac{1}{k}}\left(\sup _{f \in B}\left\|\frac{\hat{d}^{j} f(0)}{j !}\right\|_{\Theta}\right)^{\frac{1}{j}} \leq \rho .
$$

For $k=+\infty$ and $A \in(0,+\infty]$, a subset $B$ of $\operatorname{Exp}_{\Theta, A}^{\infty}(E)$ is bounded if, and only if, there is $\rho \in(0, A)$ such that

$$
\limsup _{j \rightarrow \infty}\left(\sup _{f \in B}\left\|\frac{\hat{d}^{j} f(0)}{j !}\right\|_{\Theta}\right)^{\frac{1}{j}} \leq \rho .
$$

Proof. Let $k \in[1,+\infty)$ and $B$ be a bounded subset of $\operatorname{Exp}_{\Theta, A}^{k}(E)$. By Grothendieck [5, p. 171, Proposition 5], $B$ is contained in the closure of a bounded subset of some $\mathscr{B}_{\Theta, \rho_{n}}^{k}(E)$, since $\operatorname{Exp}_{\Theta, A}^{k}(E)$ is the inductive limit of a sequence of $D F$-spaces of type $\mathscr{B}_{\Theta, \rho_{n}}^{k}(E)$, where $\left(\rho_{n}\right)_{n=1}^{\infty}$ is a strictly increasing sequence of positive real numbers converging to $A$. Without loss of generality we suppose that $B$ is contained in the closed unit ball of $\mathscr{B}_{\Theta, \rho}^{k}(E)$, for some $\rho \in(0, A)$. Now to get our result it is enough to show that the clousure, for the topology in $\operatorname{Exp}_{\Theta, A}^{k}(E)$, of this ball is contained in a ball of some $\mathscr{B}_{\Theta, \delta}^{k}(E)$, for some $\delta \in(0, A)$. In fact, if this is true we have

$$
\sup _{f \in B}\|f\|_{\Theta, k, \delta} \leq M,
$$

for some $M>0$ and so

$$
\delta^{-j}\left(\frac{j}{k e}\right)^{\frac{j}{k}} \sup _{f \in B}\left\|\frac{\hat{d}^{j} f(0)}{j !}\right\|_{\Theta} \leq M,
$$

for all $j \in \mathrm{N}_{0}$. Therefore,

$$
\limsup _{j \rightarrow \infty}\left(\frac{j}{k e}\right)^{\frac{1}{k}}\left(\sup _{f \in B}\left\|\frac{\hat{d}^{j} f(0)}{j !}\right\|_{\Theta}\right)^{\frac{1}{j}} \leq \delta,
$$

and (7) follows.

Let $B_{\mathscr{B}_{\Theta, \rho}^{k}(E)}=\left\{g \in \mathscr{B}_{\Theta, \rho}^{k}(E) ;\|g\|_{\Theta, k, \rho} \leq 1\right\}$ be the unit ball of $\mathscr{B}_{\Theta, \rho}^{k}(E)$. If $f$ belongs to $\overline{B_{\mathscr{B}_{\Theta, \rho}^{k}(E)}}$ (closure of $B_{\mathscr{B}_{\Theta, \rho}^{k}(E)}$ in $\operatorname{Exp}_{\Theta, A}^{k}(E)$ ), then there is a net $\left(f_{i}\right)_{i \in I}$ in $B_{\mathscr{B}_{\Theta, \rho}^{k}(E)}$ converging to $f$ in the topology of $\operatorname{Exp}_{\Theta, A}^{k}(E)$. Since $\left\|f_{i}\right\|_{\Theta, k, \rho} \leq 1$, we have

$$
\rho^{-j}\left(\frac{j}{k e}\right)^{\frac{j}{k}}\left\|\frac{\hat{d}^{j} f_{i}(0)}{j !}\right\|_{\Theta} \leq 1,
$$


for all $j \in \mathrm{N}_{0}$ and for all $i \in I$. Now, for each $j \in \mathrm{N}_{0}$, Proposition 3.1 ensures that the seminorm $p_{\Theta, k, \alpha}$, given by

$$
p_{\Theta, k, \alpha}(g)=\rho^{-j}\left(\frac{j}{k e}\right)^{\frac{j}{k}}\left\|\frac{\hat{d}^{j} g(0)}{j !}\right\|_{\Theta}
$$

where $\alpha_{j}=\rho^{-j}$ and $\alpha_{l}=0$, for $l \neq j$, is continuous in $\operatorname{Exp}_{\Theta, A}^{k}(E)$. Since $p_{\Theta, k, \alpha}\left(f_{i}\right)$ converges to $p_{\Theta, k, \alpha}(f)$, it follows from (9) that

$$
\rho^{-j}\left(\frac{j}{k e}\right)^{\frac{j}{k}}\left\|\frac{\hat{d}^{j} f(0)}{j !}\right\|_{\Theta} \leq 1,
$$

for all $j \in \mathrm{N}_{0}$. Since $f$ was chosen arbitrarily, we have

$$
\left(\frac{j}{k e}\right)^{\frac{j}{k}} \sup _{f \in \overline{B_{\mathscr{B}_{\Theta}^{k}}^{k}(E)}}\left\|\frac{\hat{d}^{j} f(0)}{j !}\right\|_{\Theta} \leq \rho^{j},
$$

for all $j \in \mathrm{N}_{0}$. Hence, if $\delta \in(\rho, A)$ we have

$$
\sup _{f \in B}\|f\|_{\Theta, k, \delta} \leq \sum_{j=0}^{\infty} \frac{1}{\delta^{j}}\left(\frac{j}{k e}\right)^{\frac{j}{k}} \sup _{f \in B}\left\|\frac{\hat{d}^{j} f(0)}{j !}\right\|_{\Theta} \leq \sum_{j=0}^{\infty}\left(\frac{\rho}{\delta}\right)^{j}=\frac{1}{1-\frac{\rho}{\delta}} .
$$

The case $k=+\infty$ is analogous.

Now suppose that (7) and (8) hold. It is a known result of the theory of topological vector spaces that a subset $L$ of a locally convex space $X$ is bounded, if and only if, each continuous seminorm on $X$ is bounded on $L$ (see Grothendieck [5, p. 25]). We use this result to prove that $B$ is bounded. Let $k \in[1,+\infty]$ and $p$ be a continuous seminorm. Then, there is $C>0$ such that

$$
p(f) \leq C\|f\|_{\Theta, k, \rho},
$$

for all $f \in \operatorname{Exp}_{\Theta, A}^{k}(E)$, that is

$$
\sup _{f \in B} p(f) \leq C \sup _{f \in B}\|f\|_{\Theta, k, \rho}
$$

Thus, we have from (7) that

$$
\sup _{f \in B}\|f\|_{\Theta, k, \rho} \leq \sum_{j=0}^{\infty} \frac{1}{\rho^{j}}\left(\frac{j}{k e}\right)^{\frac{j}{k}} \sup _{f \in B}\left\|\frac{\hat{d}^{j} f(0)}{j !}\right\|_{\Theta}<+\infty,
$$


when $k \in[1,+\infty)$, and from (8) that

$$
\sup _{f \in B}\|f\|_{\Theta, k, \rho} \leq \sum_{j=0}^{\infty} \frac{1}{\rho^{j}} \sup _{f \in B}\left\|\frac{\hat{d}^{j} f(0)}{j !}\right\|_{\Theta}<+\infty,
$$

when $k=+\infty$. Hence, $B$ is bounded in $\operatorname{Exp}_{\Theta, A}^{k}(E)$.

Corollary 3.3. For $k \in[1,+\infty]$ and $A \in(0,+\infty]$, a subset $B$ of $\operatorname{Exp}_{\Theta, A}^{k}(E)$ is bounded if, and only if, there is $\rho \in(0, A)$ such that $B$ is contained and bounded in $\mathscr{B}_{\Theta, \rho}^{k}(E)$.

Proof. It follows immediately from Proposition 3.2.

Proposition 3.4. For $k \in[1,+\infty)$ and $A \in[0,+\infty)$, a subset $B$ of $\operatorname{Exp}_{\Theta, 0, A}^{k}(E)$ is bounded if, and only if,

$$
\limsup _{j \rightarrow \infty}\left(\frac{j}{k e}\right)^{\frac{1}{k}}\left(\sup _{f \in B}\left\|\frac{\hat{d}^{j} f(0)}{j !}\right\|_{\Theta}\right)^{\frac{1}{j}} \leq A .
$$

For $k=+\infty$ and $A \in[0,+\infty)$, a subset $B$ of $\operatorname{Exp}_{\Theta, 0, A}^{\infty}(E)$ is bounded if, and only if,

$$
\limsup _{j \rightarrow \infty}\left(\sup _{f \in B}\left\|\frac{\hat{d}^{j} f(0)}{j !}\right\|_{\Theta}\right)^{\frac{1}{j}} \leq A .
$$

Proof. Let $k \in[1,+\infty)$ and $B$ be a bounded subset of $\operatorname{Exp}_{\Theta, 0, A}^{k}(E)$. Then $B$ is bounded in $\mathscr{B}_{\Theta, \rho}^{k}(E)$, for all $\rho>A$ (see Grothendieck [5, p. 24, Proposition 11]). Thus,

$$
\limsup _{j \rightarrow \infty}\left(\frac{j}{k e}\right)^{\frac{1}{k}}\left(\sup _{f \in B}\left\|\frac{\hat{d}^{j} f(0)}{j !}\right\|_{\Theta}\right)^{\frac{1}{j}} \leq A .
$$

The case $k=+\infty$ is analogous.

Now, suppose that (11) holds. Then for each $\varepsilon>0$, there is $C(\varepsilon)>0$ such that

$$
\left(\frac{j}{k e}\right)^{\frac{j}{k}} \sup _{f \in B}\left\|\frac{\hat{d}^{j} f(0)}{j !}\right\|_{\Theta} \leq C(\varepsilon)(A+\varepsilon)^{j},
$$

for all $j \in \mathrm{N}_{0}$. If $\rho>A$, we have

$$
\rho^{-j}\left(\frac{j}{k e}\right)^{\frac{j}{k}} \sup _{f \in B}\left\|\frac{\hat{d}^{j} f(0)}{j !}\right\|_{\Theta} \leq C(\varepsilon)\left(\frac{A+\varepsilon}{\rho}\right)^{j},
$$


for all $j \in \mathrm{N}_{0}$. Choose $\varepsilon>0$ be such that $\rho>A+\varepsilon$. Thus

$$
\begin{aligned}
\sup _{f \in B}\|f\|_{\Theta, k, \rho} & \leq \sum_{j=0}^{\infty} \frac{1}{\rho^{j}}\left(\frac{j}{k e}\right)^{\frac{j}{k}} \sup _{f \in B}\left\|\frac{\hat{d}^{j} f(0)}{j !}\right\|_{\Theta} \\
& \leq C(\varepsilon) \sum_{j=0}^{\infty}\left(\frac{A+\varepsilon}{\rho}\right)^{j}<+\infty .
\end{aligned}
$$

Hence, $B$ is bounded in $\mathscr{B}_{\Theta, \rho}^{k}(E)$ for each $\rho>A$, and so $B$ is bounded in $\operatorname{Exp}_{\Theta, 0, A}^{k}(E)$,

\section{Fourier-Borel Transforms: Main Results}

We start this section recalling the isomorphism given by Borel transform according to [3].

Let $\Theta$ be a $\pi_{1}$-holomorphy type from $E$ to $F$. We define the Borel transform

$$
\mathscr{B}_{\Theta}:\left[\mathscr{P}_{\Theta}\left({ }^{j} E ; F\right)\right]^{\prime} \rightarrow \mathscr{P}\left({ }^{j} E^{\prime} ; F^{\prime}\right)
$$

by $\mathscr{B}_{\Theta} T(\varphi)(y)=T\left(\varphi^{j} y\right)$, for all $\left.T \in\left[\mathscr{P}_{\Theta}{ }^{j} E ; F\right)\right]^{\prime}, \varphi \in E^{\prime}$ and $y \in F$. It is clear that $\mathscr{B}_{\Theta}$ is well-defined and linear. By condition (1) of Definition 2.14, we have that $\mathscr{B}_{\Theta}$ is continuous and $\left\|\mathscr{B}_{\Theta} T\right\| \leq\|T\|$. And by condition (2) we have that $\mathscr{B}_{\Theta}$ is injective.

We denote by $\mathscr{P}_{\Theta^{\prime}}\left({ }^{j} E^{\prime} ; F^{\prime}\right)$ the range of $\mathscr{B}_{\Theta}$ in $\mathscr{P}\left({ }^{j} E^{\prime} ; F^{\prime}\right)$ and define a norm on $\left.\mathscr{P}_{\Theta^{\prime}}{ }^{j} E^{\prime} ; F^{\prime}\right)$ by $\left\|\mathscr{B}_{\Theta} T\right\|_{\Theta^{\prime}}=\|T\|$. Thus $\left(\left[\mathscr{P}_{\Theta}\left({ }^{j} E ; F\right)\right]^{\prime},\|\cdot\|\right)$ is isometrically isomorphic to $\left(\mathscr{P}_{\Theta^{\prime}}\left({ }^{j} E^{\prime} ; F^{\prime}\right),\|\cdot\|_{\Theta^{\prime}}\right)$.

The next result explains why we use the norm $\|\cdot\|_{\Theta^{\prime}}$ instead of the usual sup norm on $\mathscr{P}\left({ }^{j} E^{\prime} ; F^{\prime}\right)$.

Proposition 4.1 ([3, Proposition 2.6]). Let $\left(\mathscr{P}_{\Theta}\left({ }^{j} E ; F\right)\right)_{j=0}^{\infty}$ be a $\pi_{1-}$ holomorphy type from $E$ to $F$. If the Borel transform

$$
\mathscr{B}_{\Theta}:\left(\left[\mathscr{P}_{\Theta}\left({ }^{j} E ; F\right)\right]^{\prime},\|\cdot\|\right) \rightarrow\left(\mathscr{P}\left({ }^{j} E^{\prime} ; F^{\prime}\right),\|\cdot\|\right)
$$

is a topological isomorphism onto its range, then $\mathscr{P}_{N}\left({ }^{j} E ; F\right)=\mathscr{P}_{\Theta}\left({ }^{j} E ; F\right)$ as sets, and the identity mapping $\mathscr{P}_{N}\left({ }^{j} E ; F\right) \rightarrow \mathscr{P}_{\Theta}\left({ }^{j} E ; F\right)$ is a topological isomorphism, where $\mathscr{P}_{N}\left({ }^{j} E ; F\right)$ denotes the space of all $j$-homogeneous nuclear polynomials from $E$ to $F$.

Here we are considering the usual norm on $\mathscr{P}\left({ }^{j} E^{\prime} ; F^{\prime}\right)$.

The next result ensures that the inclusion $\mathscr{P}_{\Theta^{\prime}}\left({ }^{j} E^{\prime} ; F^{\prime}\right) \hookrightarrow \mathscr{P}\left({ }^{j} E^{\prime} ; F^{\prime}\right)$ is continuous. 
Proposition 4.2. If $\left(\mathscr{P}_{\Theta}\left({ }^{j} E ; F\right)\right)_{j=0}^{\infty}$ is a $\pi_{1}$-holomorphy type from $E$ to $\mathrm{C}$, then for each $j \in \mathrm{N}_{0}$, the inclusion $\mathscr{P}_{\Theta^{\prime}}\left({ }^{j} E^{\prime} ; F^{\prime}\right) \subset \mathscr{P}\left({ }^{j} E^{\prime} ; F^{\prime}\right)$ is continuous and $\left\|\mathscr{B}_{\Theta} T\right\| \leq\left\|\mathscr{B}_{\Theta} T\right\|_{\Theta^{\prime}}$, for all $T \in\left[\mathscr{P}_{\Theta}\left({ }^{j} E ; F\right)\right]^{\prime}$.

PRoof. It is enough to see that

$$
\left\|\mathscr{B}_{\Theta} T\right\|=\sup _{\|\phi\| \leq 1} \sup _{\|y\| \leq 1}\left|T\left(\phi^{m} y\right)\right| \leq\|T\|=\left\|\mathscr{B}_{\Theta} T\right\|_{\Theta^{\prime}} .
$$

Definition 4.3. Let $\left(\mathscr{P}_{\Theta}\left({ }^{j} E\right)\right)_{j=0}^{\infty}$ be a $\pi_{1}$-holomorphy type from $E$ to $C$. If $\rho>0$ and $k \geq 1$, we denote by $\mathscr{B}_{\Theta^{\prime}, \rho}^{k}\left(E^{\prime}\right)$ the complex vector space of all $f \in \mathscr{H}\left(E^{\prime}\right)$ such that $\hat{d}^{j} f(0) \in \mathscr{P}_{\Theta^{\prime}}\left({ }^{j} E^{\prime}\right)$, for all $j \in \mathrm{N}_{0}$ and

$$
\|f\|_{\Theta^{\prime}, k, \rho}=\sum_{j=0}^{\infty} \rho^{-j}\left(\frac{j}{k e}\right)^{\frac{j}{k}}\left\|\frac{1}{j !} \hat{d}^{j} f(0)\right\|_{\Theta^{\prime}}<+\infty .
$$

A simple adaptation of the proof of Proposition 2.3, using that the inclusion $\mathscr{P}_{\Theta^{\prime}}\left({ }^{j} E^{\prime} ; F^{\prime}\right) \hookrightarrow \mathscr{P}\left({ }^{j} E^{\prime} ; F^{\prime}\right)$ is continuous via Proposition 4.2, guarantees that $\|\cdot\|_{\Theta^{\prime}, k, \rho}$ is a complete norm on $\mathscr{B}_{\Theta^{\prime}, \rho}^{k}\left(E^{\prime}\right)$.

For $k \geq 1$, replace the spaces $\left.\mathscr{P}_{\Theta}\left({ }^{j} E\right)\right)$ and $\mathscr{B}_{\Theta, \rho}^{k}(E)$ by the spaces $\mathscr{P}_{\Theta^{\prime}}\left({ }^{j} E^{\prime}\right)$ and $\mathscr{B}_{\Theta^{\prime}, \rho}^{k}(E)$, for all $j \in \mathrm{N}_{0}$, in Definition 2.4 to define the spaces $\operatorname{Exp}_{\Theta^{\prime}, A}^{k}\left(E^{\prime}\right)$, if $A \in(0,+\infty]$, and $\operatorname{Exp}_{\Theta^{\prime}, 0, A}^{k}\left(E^{\prime}\right)$, if $A \in[0,+\infty)$.

For $k=+\infty$, do the same with Definitions 2.8, 2.10 and 2.11 to define the spaces $\operatorname{Exp}_{\Theta^{\prime}, A}^{\infty}\left(E^{\prime}\right)$, if $A \in(0,+\infty]$, and $\operatorname{Exp}_{\Theta^{\prime}, 0, A}^{\infty}\left(E^{\prime}\right)$, if $A \in[0,+\infty)$.

Analogues of Propositions 2.5, 2.7, 2.12 and 2.13 for the spaces we have just defined hold with identical proofs (modulo the use of Proposition 4.2). The analogous of Proposition 2.5 shall be refered to as Propositions 2.5'.

Now we introduce the Fourier-Borel transform:

Definition 4.4. Let $\left(\mathscr{P}_{\Theta}\left({ }^{j} E\right)\right)_{j=0}^{\infty}$ be a $\pi_{1}$-holomorphy type from $E$ to C. For $k \in(1,+\infty]$ and $A \in(0,+\infty]$, the Fourier-Borel transform $\mathscr{F} T$ of $T \in\left[\operatorname{Exp}_{\Theta, A}^{k}(E)\right]^{\prime}$ is the function on $E^{\prime}$ defined by $\mathscr{F} T(\varphi)=T\left(e^{\varphi}\right) \in \mathrm{C}$.

For $k \in(1,+\infty]$ and $A \in[0,+\infty)$, the Fourier-Borel transform $\mathscr{F} T$ of $T \in\left[\operatorname{Exp}_{\Theta, 0, A}^{k}(E)\right]^{\prime}$ is the function on $E^{\prime}$ defined by $\mathscr{F} T(\varphi)=T\left(e^{\varphi}\right) \in \mathrm{C}$.

For $k=1$ and $A \in(0,+\infty]$, the Fourier-Borel transform $\mathscr{F} T$ of $T \in$ $\left[\operatorname{Exp}_{\Theta, A}^{1}(E)\right]^{\prime}$ is the function on $B_{A}(0) \subset E^{\prime}$ defined by $\mathscr{F} T(\varphi)=T\left(e^{\varphi}\right) \in \mathrm{C}$.

In all cases, Proposition 2.15 ensures that $\mathscr{F} T$ is well-defined.

As usual we set $A^{-1}=\frac{1}{A}$, for $A \in(0,+\infty)$. If $A=0$, we set $A^{-1}=+\infty$ and if $A=+\infty$, we set $A^{-1}=0$. For $k \in(1,+\infty)$, we denote by $k^{\prime}$ its conjugate, that is, $\frac{1}{k}+\frac{1}{k^{\prime}}=1$. For $k=1$, we set $k^{\prime}=+\infty$ and for 
$k=+\infty$, we set $k^{\prime}=1$. We define $\lambda(k)=\frac{k}{(k-1)^{\frac{k-1}{k}}}$, for $k \in(1,+\infty)$. Since $\lim _{k \rightarrow 1} \lambda(k)=1=\lim _{k \rightarrow \infty} \lambda(k)$, we set $\lambda(1)=\lambda(\infty)=1$.

THEOREM 4.5. If $\left(\mathscr{P}_{\Theta}\left({ }^{j} E\right)\right)_{j=0}^{\infty}$ is a $\pi_{1}$-holomorphy type from $E$ to $\mathrm{C}$, then the mapping

$$
\mathscr{F}:\left[\operatorname{Exp}_{\Theta, A}^{k}(E)\right]^{\prime} \longrightarrow \operatorname{Exp}_{\Theta^{\prime}, 0,(\lambda(k) A)^{-1}}^{k^{\prime}}\left(E^{\prime}\right),
$$

given by $\mathscr{F} T(\varphi)=T\left(e^{\varphi}\right)$, for all $T \in\left[\operatorname{Exp}_{\Theta, A}^{k}(E)\right]^{\prime}$, with $\varphi \in E^{\prime}$ and $k \in[1,+\infty]$, establishes an algebraic isomorphism between these spaces, for all $A \in(0,+\infty]$.

Proof. First we consider $k \in(1,+\infty)$ and let us prove that $\mathscr{F}$ is welldefined. Let $T \in\left[\operatorname{Exp}_{\Theta, A}^{k}(E)\right]^{\prime}$, then for each $\rho \in(0, A)$ there is $C(\rho)>0$ such that

$$
|T(f)| \leq C(\rho)\|f\|_{\Theta, k, \rho}=C(\rho) \sum_{j=0}^{\infty} \rho^{-j}\left(\frac{j}{k e}\right)^{\frac{j}{k}}\left\|\frac{1}{j !} \hat{d}^{j} f(0)\right\|_{\Theta},
$$

for all $f \in \operatorname{Exp}_{\Theta, A}^{k}(E)$. Thus, for $P \in \mathscr{P}_{\Theta}\left({ }^{j} E\right)$ we have

$$
|T(P)| \leq C(\rho) \rho^{-j}\left(\frac{j}{k e}\right)^{\frac{j}{k}}\|P\|_{\Theta} .
$$

For $T_{j}=\left.T\right|_{\left.\mathscr{P}_{\Theta}{ }^{j} E\right)}$, it follows from the definition of the Borel transform that $\left.\mathscr{B}_{\Theta} T_{j} \in \mathscr{P}_{\Theta^{\prime}}{ }^{j} E^{\prime}\right)$, where $\mathscr{B}_{\Theta} T_{j}(\varphi)=T_{j}\left(\varphi^{j}\right)$, for all $\varphi \in E^{\prime}$ and $\left\|T_{j}\right\|=$ $\left\|\mathscr{B}_{\Theta} T_{j}\right\|_{\Theta^{\prime}}$. Hence, it follows from (13) that

$$
\left\|\mathscr{B}_{\Theta} T_{j}\right\|_{\Theta^{\prime}}=\left\|T_{j}\right\| \leq C(\rho) \rho^{-j}\left(\frac{j}{k e}\right)^{\frac{j}{k}}
$$

for each $\rho \in(0, A)$, and we may write

$$
\mathscr{F} T(\varphi)=T\left(e^{\varphi}\right)=\sum_{j=0}^{\infty} \frac{1}{j !} T\left(\varphi^{j}\right)=\sum_{j=0}^{\infty} \frac{1}{j !} \mathscr{B}_{\Theta} T_{j}(\varphi)
$$


for all $\varphi \in E^{\prime}$. By (14) we have

$$
\begin{aligned}
& \limsup _{j \rightarrow \infty}\left(\frac{j}{k^{\prime} e}\right)^{\frac{1}{k^{\prime}}} \frac{1}{(j !)^{\frac{1}{j}}}\left\|\mathscr{B}_{\Theta} T_{j}\right\|_{\Theta^{\prime}}^{\frac{1}{j}} \\
& \quad \leq \limsup _{j \rightarrow \infty}(C(\rho))^{\frac{1}{j}} \frac{1}{\rho}\left(\frac{j}{k e}\right)^{\frac{1}{k}}\left(\frac{j}{k^{\prime} e}\right)^{\frac{1}{k^{\prime}}}\left(\frac{1}{j !}\right)^{\frac{1}{j}} \\
& \quad=\frac{1}{\rho}\left(\frac{1}{k}\right)^{\frac{1}{k}}\left(\frac{1}{k^{\prime}}\right)^{\frac{1}{k^{\prime}}}=\frac{1}{\rho \lambda(k)},
\end{aligned}
$$

for all $\rho \in(0, A)$. Hence

$$
\limsup _{j \rightarrow \infty}\left(\frac{j}{k^{\prime} e}\right)^{\frac{1}{k^{\prime}}}\left(\frac{1}{j !}\right)^{\frac{1}{j}}\left\|\mathscr{B}_{\Theta} T_{j}\right\|_{\Theta^{\prime}}^{\frac{1}{j}} \leq \frac{1}{A \lambda(k)}<+\infty
$$

and so

$$
\limsup _{j \rightarrow \infty}\left(\frac{1}{j !}\right)^{\frac{1}{j}}\left\|\mathscr{B}_{\Theta} T_{j}\right\|_{\Theta^{\prime}}^{\frac{1}{j}}=0
$$

since

$$
\limsup _{j \rightarrow \infty}\left(\frac{j}{k^{\prime} e}\right)^{\frac{1}{k^{\prime}}}=+\infty .
$$

By Proposition 4.2, we have $\left\|\mathscr{B}_{\Theta} T_{j}\right\| \leq\left\|\mathscr{B}_{\Theta} T_{j}\right\|_{\Theta^{\prime}}$. Then the radius of convergence of (15) is $+\infty$ and Proposition $2.5^{\prime}$ ensures that $\mathscr{F} T \in \operatorname{Exp}_{\Theta^{\prime}, 0,(\lambda(k) A)^{-1}}^{k^{\prime}}\left(E^{\prime}\right)$.

Now we consider $H \in \operatorname{Exp}_{\Theta^{\prime}, 0,(\lambda(k) A)^{-1}}^{k^{\prime}}\left(E^{\prime}\right)$. Hence for each $\rho \in(0, A)$, there is $C(\rho)>0$ such that

$$
\left(\frac{j}{k^{\prime} e}\right)^{\frac{j}{k^{\prime}}} \frac{1}{j !}\left\|\hat{d}^{j} H(0)\right\|_{\Theta^{\prime}} \leq C(\rho) \frac{1}{(\rho \lambda(k))^{j}}
$$

for all $j \in \mathrm{N}$. By definition of Borel transform there is $T_{j} \in\left[\mathscr{P}_{\Theta}\left({ }^{j} E\right)\right]^{\prime}$ such that $\mathscr{B}_{\Theta} T_{j}=\hat{d}^{j} H(0),\left\|T_{j}\right\|=\left\|\hat{d}^{j} H(0)\right\|_{\Theta^{\prime}}$. Then

$$
\frac{1}{j !}\left\|T_{j}\right\| \leq C(\rho) \frac{1}{\rho^{j}} \frac{1}{(\lambda(k))^{j}}\left(\frac{k^{\prime} e}{j}\right)^{\frac{j}{k^{\prime}}} .
$$

For $f \in \operatorname{Exp}_{\Theta, A}^{k}(E)$ we define

$$
T_{H}(f)=\sum_{j=0}^{\infty} \frac{1}{j !} T_{j}\left(\hat{d}^{j} f(0)\right) .
$$


Hence, using (16), we have

$$
\begin{aligned}
\frac{1}{j !}\left\|T_{j}\right\| & \left\|\hat{d}^{j} f(0)\right\|_{\Theta} \\
& \leq C(\rho) \frac{1}{\rho^{j}} \frac{1}{(\lambda(k))^{j}}\left(\frac{k^{\prime} e}{j}\right)^{\frac{j}{k^{\prime}}}\left\|\hat{d}^{j} f(0)\right\|_{\Theta} \\
& =C(\rho) \frac{1}{\rho^{j}}\left(\frac{1}{k}\right)^{\frac{j}{k}}\left(\frac{1}{k^{\prime}}\right)^{\frac{j}{k^{\prime}}}\left(\frac{k^{\prime} e}{j}\right)^{\frac{j}{k^{\prime}}}\left\|\hat{d}^{j} f(0)\right\|_{\Theta} \\
& =C(\rho) \frac{1}{\rho^{j}}\left(\frac{1}{k}\right)^{\frac{j}{k}}\left(\frac{e}{j}\right)^{j-\frac{j}{k}}\left\|\hat{d}^{j} f(0)\right\|_{\Theta} \\
& =C(\rho) \frac{1}{\rho^{j}}\left(\frac{j}{k e}\right)^{\frac{j}{k}}\left(\frac{e}{j}\right)^{j}\left\|\hat{d}^{j} f(0)\right\|_{\Theta} .
\end{aligned}
$$

Since $\lim _{j \rightarrow \infty} \frac{e}{j}(j !)^{\frac{1}{j}}=1$, for each $\varepsilon>0$, there is $D(\varepsilon)>0$ such that

$$
\left(\frac{e}{j}\right)^{j} \leq D(\varepsilon)(1+\varepsilon)^{j} \frac{1}{j !}
$$

for all $j \in \mathrm{N}$. Hence, it follows from (17) that

$$
\frac{1}{j !}\left\|T_{j}\right\|\left\|\hat{d}^{j} f(0)\right\|_{\Theta} \leq C(\rho) D(\varepsilon)\left(\frac{1+\varepsilon}{\rho}\right)^{j}\left(\frac{j}{k e}\right)^{\frac{j}{k}}\left\|\frac{\hat{d}^{j} f(0)}{j !}\right\|_{\Theta} .
$$

By definition of $T_{H}$ we have

$$
\begin{aligned}
\left|T_{H}(f)\right| & \leq C(\rho) D(\varepsilon) \sum_{j=0}^{\infty}\left(\frac{\rho}{1+\varepsilon}\right)^{-j}\left(\frac{j}{k e}\right)^{\frac{j}{k}}\left\|\frac{\hat{d}^{j} f(0)}{j !}\right\|_{\Theta} \\
& =C(\rho) D(\varepsilon)\|f\|_{\Theta, k, \frac{\rho}{1+\varepsilon}}
\end{aligned}
$$

for all $f \in \operatorname{Exp}_{\Theta, A}^{k}(E), \varepsilon>0$ and $\rho \in(0, A)$. Therefore $T_{H} \in\left[\operatorname{Exp}_{\Theta, A}^{k}(E)\right]^{\prime}$ and it is easy to see that $\mathscr{F} T_{H}=H$.

It is clear that $\mathscr{F}$ is linear. For the injectivity, let $T \in\left[\operatorname{Exp}_{\Theta, A}^{k}(E)\right]^{\prime}$ such that $\mathscr{F} T=0$. Then, for each $\varphi \in E^{\prime}, 0=\mathscr{F} T(\varphi)=T\left(e^{\varphi}\right)$. By Proposition 2.16(a.1) we have $T(f)=0$, for all $f \in \operatorname{Exp}_{\Theta, A}^{k}(E)$, and so $T=0$.

Now, we prove the case $k=1$. Proceeding as done before and using the same notations that we used in case $k \in(1,+\infty)$ we conclude that

$$
\mathscr{F} T(\varphi)=T\left(e^{\varphi}\right)=\sum_{j=0}^{\infty} \frac{1}{j !} T\left(\varphi^{j}\right)=\sum_{j=0}^{\infty} \frac{1}{j !} \mathscr{B}_{\Theta} T_{j}(\varphi)
$$


for all $\varphi \in E^{\prime}$ such that $\|\varphi\|<A$ and

$$
\limsup _{j \rightarrow \infty}\left\|\frac{1}{j !} \mathscr{B}_{\Theta} T_{j}\right\|_{\Theta^{\prime}}^{\frac{1}{j}} \leq \frac{1}{A} .
$$

By Proposition 4.2, we have

$$
\limsup _{j \rightarrow \infty}\left\|\frac{1}{j !} \mathscr{B}_{\Theta} T_{j}\right\|^{\frac{1}{j}} \leq \frac{1}{A}
$$

and it follows from definition of $\mathscr{H}_{\Theta^{\prime} b}\left(B_{A}(0)\right)$ that $\mathscr{F} T \in \mathscr{H}_{\Theta^{\prime} b}\left(B_{A}(0)\right)=$ $\operatorname{Exp}_{\Theta^{\prime}, 0, \frac{1}{A}}^{\infty}\left(E^{\prime}\right)$ (here $B_{A}(0)$ is the open ball in $E^{\prime}$ ). Hence $\mathscr{F}$ is well-defined.

Now we consider $H \in \operatorname{Exp}_{\Theta^{\prime}, 0, \frac{1}{A}}^{\infty}\left(E^{\prime}\right)$. Hence

$$
\limsup _{j \rightarrow \infty}\left\|\frac{\hat{d}^{j} H(0)}{j !}\right\|_{\Theta^{\prime}}^{\frac{1}{j}} \leq \frac{1}{A} .
$$

By definition of Borel transform there is $T_{j} \in\left[\mathscr{P}_{\Theta}\left({ }^{j} E\right)\right]^{\prime}$ such that $\mathscr{B}_{\Theta} T_{j}=$ $\hat{d}^{j} H(0)$ and $\left\|T_{j}\right\|=\left\|\hat{d}^{j} H(0)\right\|_{\Theta^{\prime}}$. For $f \in \operatorname{Exp}_{\Theta, A}^{1}(E)$, we define

$$
T_{H}(f)=\sum_{j=0}^{\infty} \frac{1}{j !} T_{j}\left(\hat{d}^{j} f(0)\right) .
$$

Then

$$
\left|\frac{1}{j !} T_{j}\left(\hat{d}^{j} f(0)\right)\right| \leq \frac{1}{j !}\left\|T_{j}\right\|\left\|\hat{d}^{j} f(0)\right\|_{\Theta}=\frac{1}{j !}\left\|\hat{d}^{j} H(0)\right\|_{\Theta^{\prime}}\left\|\hat{d}^{j} f(0)\right\|_{\Theta} .
$$

By (18), for each $\rho \in(0, A)$, there is $C(\rho)>0$ such that

$$
\frac{1}{j !}\left\|\hat{d}^{j} H(0)\right\|_{\Theta^{\prime}} \leq C(\rho) \frac{1}{\rho^{j}},
$$

for all $j \in \mathrm{N}_{0}$. Hence

$$
\left|T_{H}(f)\right| \leq \sum_{j=0}^{\infty} C(\rho) \frac{1}{\rho^{j}}\left\|\hat{d}^{j} f(0)\right\|_{\Theta}=C(\rho)\|f\|_{\Theta, \rho},
$$

for each $\rho \in(0, A)$ and $f \in \operatorname{Exp}_{\Theta, A}^{1}(E)$. Therefore $T_{H} \in\left[\operatorname{Exp}_{\Theta, A}^{1}(E)\right]^{\prime}$ and it is easy to see that $\mathscr{F} T_{H}=H$. The linearity is clear and the injectivity follows using Proposition 2.16(b). 
Now, we prove the case $k=+\infty$. Proceeding as before and using the same notations, we have that for $T \in\left[\operatorname{Exp}_{\Theta, A}^{\infty}(E)\right]^{\prime}=\left[\mathscr{H}_{\Theta}\left(\overline{B_{\frac{1}{A}}(0)}\right)\right]^{\prime}$,

$$
\mathscr{F} T(\varphi)=T\left(e^{\varphi}\right)=\sum_{j=0}^{\infty} \frac{1}{j !} T\left(\varphi^{j}\right)=\sum_{j=0}^{\infty} \frac{1}{j !} \mathscr{B}_{\Theta} T_{j}(\varphi)
$$

for all $\varphi \in E^{\prime}$ and

$$
\limsup _{j \rightarrow \infty}\left\|\mathscr{B}_{\Theta} T_{j}\right\|_{\Theta^{\prime}}^{\frac{1}{j}}<\frac{1}{A} .
$$

Since $\lim \sup _{j \rightarrow \infty}\left(\frac{1}{j !}\right)^{\frac{1}{j}}=0$, we have

$$
\limsup _{j \rightarrow \infty}\left(\frac{1}{j !}\right)^{\frac{1}{j}}\left\|\mathscr{B}_{\Theta} T_{j}\right\|_{\Theta^{\prime}}^{\frac{1}{j}}=0 .
$$

Thus, Proposition 4.2 implies that the radius of convergence of (19) is $+\infty$ and Proposition 2.5' ensures that $\mathscr{F} T \in \operatorname{Exp}_{\Theta^{\prime}, 0, \frac{1}{A}}^{1}\left(E^{\prime}\right)$.

Now, let $H \in \operatorname{Exp}_{\Theta^{\prime}, 0, \frac{1}{A}}^{1}\left(E^{\prime}\right)$. It follows from Proposition 2.5' that

$$
\limsup _{j \rightarrow \infty}\left\|\hat{d}^{j} H(0)\right\|_{\Theta^{\prime}}^{\frac{1}{j}} \leq \frac{1}{A} .
$$

By definition of Borel transform, there is $T_{j} \in\left[\mathscr{P}_{\Theta}\left({ }^{j} E\right)\right]^{\prime}$ such that $\mathscr{B}_{\Theta} T_{j}=$ $\hat{d}^{j} H(0)$ and $\left\|T_{j}\right\|=\left\|\hat{d}^{j} H(0)\right\|_{\Theta^{\prime}}$. If we define

$$
T_{H}(f)=\sum_{j=0}^{\infty} \frac{1}{j !} T_{j}\left(\hat{d}^{j} f(0)\right),
$$

for all $f \in \operatorname{Exp}_{\Theta, A}^{\infty}(E)$, then

$$
\left|\frac{1}{j !} T_{j}\left(\hat{d}^{j} f(0)\right)\right| \leq \frac{1}{j !}\left\|T_{j}\right\|\left\|\hat{d}^{j} f(0)\right\|_{\Theta}=\frac{1}{j !}\left\|\hat{d}^{j} H(0)\right\|_{\Theta^{\prime}}\left\|\hat{d}^{j} f(0)\right\|_{\Theta} .
$$

By (20), for each $\rho \in(0, A)$, there is $C(\rho)>0$ such that

$$
\left\|\hat{d}^{j} H(0)\right\|_{\Theta^{\prime}} \leq C(\rho) \frac{1}{\rho^{j}}
$$

for all $j \in \mathrm{N}_{0}$. Thus

$$
\begin{aligned}
\left|T_{H}(f)\right| \leq \sum_{j=0}^{\infty}\left|\frac{1}{j !} T_{j}\left(\hat{d}^{j} f(0)\right)\right| & \leq \sum_{j=0}^{\infty} C(\rho) \frac{1}{j ! \rho^{j}}\left\|\hat{d}^{j} f(0)\right\|_{\Theta} \\
& =C(\rho)\|f\|_{\Theta, \infty, \rho}
\end{aligned}
$$


for each $\rho \in(0, A)$ and each $f \in \operatorname{Exp}_{\Theta, A}^{\infty}(E)$. Then $T_{H} \in\left[\operatorname{Exp}_{\Theta, A}^{\infty}(E)\right]^{\prime}$ and it is easy to see that $\mathscr{F} T_{H}=H$. It is clear that $\mathscr{F}$ is linear and by Proposition 2.16(a.1) $\mathscr{F}$ is injective.

THEOREM 4.6. If $\left(\mathscr{P}_{\Theta}\left({ }^{j} E\right)\right)_{j=0}^{\infty}$ is a $\pi_{1}$-holomorphy type from $E$ to $\mathrm{C}$, then the mapping

$$
\mathscr{F}:\left[\operatorname{Exp}_{\Theta, 0, A}^{k}(E)\right]^{\prime} \longrightarrow \operatorname{Exp}_{\Theta^{\prime},(\lambda(k) A)^{-1}}^{k^{\prime}}\left(E^{\prime}\right),
$$

given by $\mathscr{F} T(\varphi)=T\left(e^{\varphi}\right)$, for all $T \in\left[\operatorname{Exp}_{\Theta, 0, A}^{k}(E)\right]^{\prime}$ and $\varphi \in E^{\prime}$, establishes an algebraic isomorphism between these spaces, for all $k \in(1,+\infty]$ and $A \in[0,+\infty)$.

PRoOF. The proof follows in a similar way as done in Theorem 4.5.

As we saw in Proposition 2.15, $e^{\varphi} \in \operatorname{Exp}_{\Theta, 0, A}^{1}(E)$, if $\varphi \in E^{\prime}$ and $\|\varphi\| \leq A$. Hence, if $T \in\left[\operatorname{Exp}_{\Theta, 0, A}^{1}(E)\right]^{\prime}$, the natural definition for its Fourier-Borel transform $\mathscr{F} T$ would be $\mathscr{F} T(\varphi)=T\left(e^{\varphi}\right)$, for all $\varphi \in E^{\prime}$ with $\|\varphi\| \leq A$. However it can be proved that we can define $\mathscr{F} T$ for all $\varphi \in E^{\prime}$ in a "bigger" set, in such way that it agrees with the previous definition for $\varphi \in E^{\prime}$ with $\|\varphi\| \leq A$. This is ensured by Proposition 4.8.

Definition 4.7. Let $\left(\mathscr{P}_{\Theta}\left({ }^{j} E\right)\right)_{j=0}^{\infty}$ be a $\pi_{1}$-holomorphy type from $E$ to C. For $k=1$ and $A \in[0,+\infty)$, we define the Fourier-Borel transform $\mathscr{F} T$ of $T \in\left[\operatorname{Exp}_{\Theta, 0, A}^{1}(E)\right]^{\prime}$ by

$$
\mathscr{F} T(\varphi)=\sum_{j=0}^{\infty} \frac{1}{j !} \mathscr{B}_{\Theta} T_{j}(\varphi),
$$

for all $\varphi \in E^{\prime}$ such that the series converges absolutely. Here $T_{j}=\left.T\right|_{\left.\mathscr{P}_{\Theta}{ }^{j} E\right)}$, $\mathscr{B}_{\Theta} T_{j} \in P_{\Theta^{\prime}}\left({ }^{j} E^{\prime}\right)$ is given by $\mathscr{B}_{\Theta} T_{j}(\varphi)=T_{j}\left(\varphi^{j}\right)$, for all $\varphi \in E^{\prime}$, and $\left\|T_{j}\right\|=$ $\left\|\mathscr{B}_{\Theta} T_{j}\right\|_{\Theta^{\prime}}$ by definition of Borel transform.

Proposition 4.8. If $\left(\mathscr{P}_{\Theta}\left({ }^{j} E\right)\right)_{j=0}^{\infty}$ is a $\pi_{1}$-holomorphy type from $E$ to $\mathrm{C}$, $A \in[0,+\infty)$ and $T \in\left[\operatorname{Exp}_{\Theta, 0, A}^{1}(E)\right]^{\prime}$, then there is $\rho>A$ such that $\mathscr{F} T \in$ $\mathscr{H}_{\Theta^{\prime}}^{\infty}\left(B_{\rho}(0)\right)$, where $B_{\rho}(0)$ is the open ball in $E^{\prime}$.

Proof. If $T \in\left[\operatorname{Exp}_{\Theta, 0, A}^{1}(E)\right]^{\prime}$, there are $\delta>A$ and $C(\delta)>0$ such that

$$
|T(f)| \leq C(\delta)\|f\|_{\Theta, \delta}=C(\delta) \sum_{j=0}^{\infty} \delta^{-j}\left\|\hat{d}^{j} f(0)\right\|_{\Theta},
$$

for all $f \in \operatorname{Exp}_{\Theta, 0, A}^{1}(E)$. Hence

$$
|T(P)| \leq C(\delta) \delta^{-j} j !\|P\|_{\Theta}
$$


for all $P \in \mathscr{P}_{\Theta}\left({ }^{j} E\right)$. Thus

$$
\left\|\mathscr{B}_{\Theta} T_{j}\right\|_{\Theta^{\prime}}=\left\|T_{j}\right\| \leq C(\delta) j ! \delta^{-j},
$$

for all $j \in \mathrm{N}_{0}$ and

$$
\underset{j \rightarrow \infty}{\limsup }\left\|\frac{\mathscr{B}_{\Theta} T_{j}}{j !}\right\|_{\Theta^{\prime}}^{\frac{1}{j}} \leq \frac{1}{\delta} .
$$

Let $\rho \in(A, \delta)$, then

$$
\limsup _{j \rightarrow \infty}\left\|\frac{\mathscr{B}_{\Theta} T_{j}}{j !}\right\|_{\Theta^{\prime}}^{\frac{1}{j}}<\frac{1}{\rho},
$$

and so $\mathscr{F} T \in \mathscr{H}\left(B_{\rho}(0)\right)$. Furthermore,

$$
\sum_{j=0}^{\infty} \rho^{j}\left\|\frac{1}{j !} \mathscr{B}_{\Theta} T_{j}\right\|_{\Theta^{\prime}}<+\infty,
$$

then $\mathscr{F} T \in \mathscr{H}_{\Theta^{\prime}}^{\infty}\left(B_{\rho}(0)\right)$.

Now we are able to prove the algebraic isomorphism of the Fourier-Borel transform for the remaining case.

THEOREM 4.9. If $\left(\mathscr{P}_{\Theta}\left({ }^{j} E\right)\right)_{j=0}^{\infty}$ is a $\pi_{1}$-holomorphy type from $E$ to $\mathrm{C}$, then the mapping

$$
\mathscr{F}: T \in\left[\operatorname{Exp}_{\Theta, 0, A}^{1}(E)\right]^{\prime} \longrightarrow \mathscr{F} T \in \operatorname{Exp}_{\Theta^{\prime}, \frac{1}{A}}^{\infty}\left(E^{\prime}\right),
$$

establishes an algebraic isomorphism between these two spaces, for $A \in$ $[0,+\infty)$. Here we are identifying the class $[\mathscr{F} T]$ with its representative $\mathscr{F} T$.

Proof. By definition of $\operatorname{Exp}_{\Theta^{\prime}, \frac{1}{A}}^{\infty}\left(E^{\prime}\right)$ and Proposition 4.8 it is clear that $\mathscr{F} T$ belongs to $\operatorname{Exp}_{\Theta^{\prime}, \frac{1}{A}}^{\infty}\left(E^{\prime}\right)$, for all $T \in\left[\operatorname{Exp}_{\Theta, 0, A}^{1}(E)\right]^{\prime}$. Now, let $T \in$ $\left[\operatorname{Exp}_{\Theta, 0, A}^{1}(E)\right]^{\prime}$ be such that $\mathscr{F} T=0$. By Proposition 4.8, there is $\rho>A$ such that $\mathscr{F} T(\varphi)=0$, for all $\varphi \in E^{\prime}$ with $\|\varphi\|<\rho$. Hence $\mathscr{B}_{\Theta} T_{j}(\varphi)=0$, for all $\varphi \in B_{\rho}(0) \subseteq E^{\prime}$ and $j \in \mathrm{N}_{0}$. Consequently, $\mathscr{B}_{\Theta} T_{j}=0,\left\|T_{j}\right\|=\left\|\mathscr{B}_{\Theta} T_{j}\right\|_{\Theta^{\prime}}=$ 0 and $\left.T\right|_{\left.\mathscr{P}_{\Theta}{ }^{j} E\right)}=0$, for all $j \in \mathrm{N}_{0}$. Hence, it follows from Proposition 2.13 that $T(f)=0$, for all $f \in \operatorname{Exp}_{\Theta, 0, A}^{1}(E)$. Therefore $T=0$ and consequently, $\mathscr{F}$ is injective. The linearity of $\mathscr{\mathscr { F }}$ is clear. Now, let $H \in \operatorname{Exp}_{\Theta^{\prime}, \frac{1}{A}}^{\infty}\left(E^{\prime}\right)$, then there is $\rho>A$ such that $H \in \mathscr{H}_{\Theta^{\prime}}^{\infty}\left(B_{\rho}(0)\right)$, Thus

$$
\limsup _{j \rightarrow \infty}\left\|\frac{\hat{d}^{j} H(0)}{j !}\right\|_{\Theta^{\prime}}^{\frac{1}{j}} \leq \frac{1}{\rho}
$$


and, for all $\varepsilon>0$, there is $C(\varepsilon)>0$ such that

$$
\frac{1}{j !}\left\|\hat{d}^{j} H(0)\right\|_{\Theta^{\prime}} \leq C(\varepsilon)\left(\frac{1+\varepsilon}{\rho}\right)^{j},
$$

for all $j \in \mathrm{N}_{0}$. By definition of Borel transform, there is $T_{j} \in\left[\mathscr{P}_{\Theta}\left({ }^{j} E\right)\right]^{\prime}$ such that $\mathscr{B}_{\Theta} T_{j}=\hat{d}^{j} H(0)$ and $\left\|T_{j}\right\|=\left\|\hat{d}^{j} H(0)\right\|_{\Theta^{\prime}}$. For $f \in \operatorname{Exp}_{\Theta, 0, A}^{1}(E)$, we define

$$
T_{H}(f)=\sum_{j=0}^{\infty} \frac{1}{j !} T_{j}\left(\hat{d}^{j} f(0)\right) .
$$

Hence

$$
\begin{aligned}
\left|T_{H}(f)\right| & \leq \sum_{j=0}^{\infty} \frac{1}{j !}\left|T_{j}\left(\hat{d}^{j} f(0)\right)\right| \leq \sum_{j=0}^{\infty} \frac{1}{j !}\left\|\hat{d}^{j} H(0)\right\|_{\Theta^{\prime}}\left\|\hat{d}^{j} f(0)\right\|_{\Theta} \\
& \leq C(\varepsilon) \sum_{j=0}^{\infty}\left(\frac{1+\varepsilon}{\rho}\right)^{j}\left\|\hat{d}^{j} f(0)\right\|_{\Theta}=C(\varepsilon)\|f\|_{\Theta, \frac{\rho}{1+\varepsilon}}
\end{aligned}
$$

for all $f \in \operatorname{Exp}_{\Theta, 0, A}^{1}(E)$ and $\varepsilon>0$, such that $\frac{\rho}{1+\varepsilon}>A$. Therefore, $T_{H} \in$ $\left[\operatorname{Exp}_{\Theta, 0, A}^{1}(E)\right]^{\prime}$ and $\mathscr{F} T_{H}=H$.

Now we are able to prove that, in some cases, the Fourier-Borel transforms are topological isomophisms.

THEOREM 4.10. If $\left(\mathscr{P}_{\Theta}\left({ }^{j} E\right)\right)_{j=0}^{\infty}$ is a $\pi_{1}$-holomorphy type from $E$ to $\mathrm{C}$, then the Fourier-Borel transform $\mathscr{F}$ is a topological isomorphism between the spaces $\left[\operatorname{Exp}_{\Theta, A}^{k}(E)\right]_{\beta}^{\prime}$ and $\operatorname{Exp}_{\Theta^{\prime}, 0,(\lambda(k) A)^{-1}}^{k^{\prime}}\left(E^{\prime}\right)$, for all $k \in[1,+\infty]$ and $A \in(0,+\infty]$.

Here $\beta$ denotes the strong topology on the dual.

Proof. By the Open Mapping Theorem it is enough to show that $\mathscr{F}^{-1}$ is continuous, because $\left[\operatorname{Exp}_{\Theta, A}^{k}(E)\right]_{\beta}^{\prime}$ and $\operatorname{Exp}_{\Theta^{\prime}, 0,(\lambda(k) A)^{-1}}^{k^{\prime}}\left(E^{\prime}\right)$ are Fréchet spaces. Note that $\left[\operatorname{Exp}_{\Theta, A}^{k}(E)\right]_{\beta}^{\prime}$ is a Fréchet space, because it is the strong dual of a $D F$-space (see Grothendieck [5, p. 166, Corollary 4]).

We will prove that $\mathscr{F}^{-1}$ is continuous showing that for each continuous seminorm $q$ in $\left[\operatorname{Exp}_{\Theta, A}^{k}(E)\right]_{\beta}^{\prime}$, there are $C>0$ and a continuous seminorm $p$ in $\operatorname{Exp}_{\Theta^{\prime}, 0,(\lambda(k) A)^{-1}}^{k^{\prime}}\left(E^{\prime}\right)$ such that $q\left(F^{-1}(H)\right)=q\left(T_{H}\right) \leq C p(H)$, for all $H \in$ $\operatorname{Exp}_{\Theta^{\prime}, 0,(\lambda(k) A)^{-1}}^{k^{\prime}}\left(E^{\prime}\right)$ (here $T_{H}$ is the same we used several times before). We know that the strong topology on the dual is generated by a family of seminorms $p_{B}(S)=\sup _{f \in B}|S(f)|$, where $S \in\left[\operatorname{Exp}_{\Theta, A}^{k}(E)\right]_{\beta}^{\prime}$ and $B \subseteq \operatorname{Exp}_{\Theta, A}^{k}(E)$ is a bounded subset. 
Let $B \in \operatorname{Exp}_{\Theta, A}^{k}(E)$ be a bounded subset, for $k \in(1,+\infty)$. By Proposition 3.2 there is $\rho \in(0, A)$ such that

$$
\limsup _{j \rightarrow \infty}\left(\frac{j}{k e}\right)^{\frac{1}{k}}\left(\sup _{f \in B}\left\|\frac{\hat{d}^{j} f(0)}{j !}\right\|_{\Theta}\right)^{\frac{1}{j}} \leq \rho .
$$

Thus, for each $\varepsilon>0$ there is $C(\varepsilon)>0$ such that

$$
\left(\frac{j}{k e}\right)^{\frac{j}{k}} \sup _{f \in B}\left\|\frac{\hat{d}^{j} f(0)}{j !}\right\|_{\Theta} \leq C(\varepsilon)(\rho+\varepsilon)^{j}
$$

for all $j \in \mathrm{N}_{0}$. Then,

$$
\begin{aligned}
\sup _{f \in B}\left|F^{-1}(H)(f)\right| & =\sup _{f \in B}\left|T_{H}(f)\right| \leq \sum_{j=0}^{\infty}\left\|\hat{d}^{j} H(0)\right\|_{\Theta^{\prime}} \sup _{f \in B}\left\|\frac{\hat{d}^{j} f(0)}{j !}\right\|_{\Theta} \\
& \leq C(\varepsilon) \sum_{j=0}^{\infty}(\rho+\varepsilon)^{j}\left(\frac{k e}{j}\right)^{\frac{j}{k}} j !\left\|\frac{\hat{d}^{j} H(0)}{j !}\right\|_{\Theta^{\prime}}
\end{aligned}
$$

Since

$$
\left(\frac{k e}{j}\right)^{\frac{j}{k}}=(\lambda(k))^{j}\left(\frac{j}{k^{\prime} e}\right)^{\frac{j}{k^{\prime}}} \frac{e^{j}}{j^{j}}
$$

we have

$$
\begin{aligned}
\sum_{j=0}^{\infty}(\rho+\varepsilon)^{j}\left(\frac{k e}{j}\right)^{\frac{j}{k}} j !\left\|\frac{\hat{d}^{j} H(0)}{j !}\right\|_{\Theta^{\prime}} \\
\leq \sum_{j=0}^{\infty}(\lambda(k))^{j}(\rho+\varepsilon)^{j} \frac{j !}{j^{j}} e^{j}\left(\frac{j}{k^{\prime} e}\right)^{\frac{j}{k^{\prime}}}\left\|\frac{\hat{d}^{j} H(0)}{j !}\right\|_{\Theta^{\prime}}
\end{aligned}
$$

and since

$$
\lim _{j \rightarrow \infty} \frac{e}{j}(j !)^{\frac{1}{j}}=1
$$

there is $D(\varepsilon)>0$ such that

$$
\frac{j !}{j^{j}} e^{j} \leq D(\varepsilon)(1+\varepsilon)^{j},
$$

for all $j \in \mathrm{N}$. Therefore

$$
\sup _{f \in B}\left|\mathscr{F}^{-1}(H)(f)\right| \leq C(\varepsilon) D(\varepsilon)\|H\|_{\Theta^{\prime}, k^{\prime}, \frac{1}{\lambda(k)(\rho+\varepsilon)(1+\varepsilon)}} .
$$


Now, choosing $\varepsilon>0$ such that $(\rho+\varepsilon)(1+\varepsilon)<A$, we have $\frac{1}{\lambda(k)(\rho+\varepsilon)(1+\varepsilon)}>$ $\frac{1}{\lambda(k) A}$ and the continuity of $\mathscr{F}^{-1}$ follows.

Proceeding the same way we have

$$
\sup _{f \in B}\left|\mathscr{F}^{-1}(H)(f)\right| \leq C(\varepsilon)\|H\|_{\Theta^{\prime}, \infty, \frac{1}{\rho+\varepsilon}},
$$

if $k=1$ and

$$
\sup _{f \in B}\left|F^{-1}(H)(f)\right| \leq C(\varepsilon)\|H\|_{\Theta^{\prime}, \frac{1}{\rho+\varepsilon}},
$$

if $k=+\infty$. Then, choosing $\varepsilon>0$ such that $(\rho+\varepsilon)<A$, we have $\frac{1}{\rho+\varepsilon}>\frac{1}{A}$ and the continuity of $F^{-1}$ follows.

It is an open problem if the Fourier-Borel transform, in the next case, is a topological isomorphism. But it is possible to prove that $\mathscr{F}^{-1}$ is continuous, as follows.

THEOREM 4.11. If $E^{\prime}$ has the $\lambda$-bounded approximation property, $k \in$ $[1,+\infty]$ and $A \in[0,+\infty)$, then

$$
\mathscr{F}^{-1}: \operatorname{Exp}_{\Theta^{\prime},(\lambda(k) A)^{-1}}^{k^{\prime}}\left(E^{\prime}\right) \rightarrow\left[\operatorname{Exp}_{\Theta, 0, A}^{k}(E)\right]_{\beta}^{\prime},
$$

is continuous. Again $\beta$ denotes the strong topology on the dual.

Proof. Let $B \in \operatorname{Exp}_{\Theta, 0, A}^{k}(E)$ be a bounded subset. By Proposition 3.4 we have

$$
\limsup _{j \rightarrow \infty}\left(\frac{j}{k e}\right)^{\frac{1}{k}}\left(\sup _{f \in B}\left\|\frac{\hat{d}^{j} f(0)}{j !}\right\|_{\Theta}\right)^{\frac{1}{j}} \leq A .
$$

For $\rho>A$ there is $C(\rho)>0$ such that

$$
\left(\frac{j}{k e}\right)^{\frac{j}{k}} \sup _{f \in B}\left\|\frac{\hat{d}^{j} f(0)}{j !}\right\|_{\Theta} \leq C(\rho) \rho^{j},
$$

for all $j \in \mathrm{N}_{0}$. Thus

$$
\begin{aligned}
\sup _{f \in B}\left|\mathscr{F}^{-1}(H)(f)\right| & \leq \sum_{j=0}^{\infty}\left\|\hat{d}^{j} H(0)\right\|_{\Theta^{\prime}} \sup _{f \in B}\left\|\frac{\hat{d}^{j} f(0)}{j !}\right\|_{\Theta} \\
& \leq C(\rho) \sum_{j=0}^{\infty} \rho^{j}\left(\frac{k e}{j}\right)^{\frac{j}{k}} j !\left\|\frac{\hat{d}^{j} H(0)}{j !}\right\|_{\Theta^{\prime}} \\
& =C(\rho) \sum_{j=0}^{\infty}(\rho \lambda(k))^{j}\left(\frac{j}{k^{\prime} e}\right)^{\frac{j}{k^{\prime}}} \frac{j !}{j^{j}} e^{j}\left\|\frac{\hat{d}^{j} H(0)}{j !}\right\|_{\Theta^{\prime}}
\end{aligned}
$$


By (23) we have

$$
\begin{aligned}
\sup _{f \in B}\left|\mathscr{F}^{-1}(H)(f)\right| & \leq C(\rho) \sum_{j=0}^{\infty}(\rho \lambda(k))^{j}\left(\frac{j}{k^{\prime} e}\right)^{\frac{j}{k^{\prime}}} \frac{j !}{j^{j}} e^{j}\left\|\frac{\hat{d}^{j} H(0)}{j !}\right\|_{\Theta^{\prime}} \\
& \leq C(\rho) D(\varepsilon) \sum_{j=0}^{\infty}(\rho(1+\varepsilon) \lambda(k))^{j}\left(\frac{j}{k^{\prime} e}\right)^{\frac{j}{k^{\prime}}}\left\|\frac{\hat{d}^{j} H(0)}{j !}\right\|_{\Theta^{\prime}} \\
& =C(\rho) D(\varepsilon)\|H\|_{\Theta^{\prime}, k^{\prime}, \frac{1}{\lambda(k) \rho(1+\varepsilon)},}
\end{aligned}
$$

for all $\varepsilon>0$ and $\rho>A$. Hence,

$$
\sup _{f \in B}\left|\mathscr{F}^{-1}(H)(f)\right| \leq C(\rho) D(\varepsilon)\|H\|_{\Theta^{\prime}, k^{\prime}, r},
$$

for all $r<\frac{1}{\lambda(k) A}$ and this prove that $\mathscr{F}^{-1}$ is continuous.

The proof for $k=1$ and $k=+\infty$ follows the same pattern.

Acknowledgement. The authors thank the referee for his/her helpful suggestions.

\section{REFERENCES}

1. Fávaro, V. V.,The Fourier-Borel transform between spaces of entire functions of a given type and order, Port. Math. 65 (2008), 285-309.

2. Fávaro, V. V., Convolution equations on spaces of quasi-nuclear functions of a given type and order, Bull. Belg. Math. Soc. Simon Stevin 17 (2010), 535-569.

3. Fávaro, V. V., Jatobá, A. M., Holomorphy types and spaces of entire functions of bounded type on Banach spaces, Czechoslovak Math. J. 59 (2009), 909-927.

4. Dineen, S., Holomorphy types on a Banach space, Studia Math. 39 (1971), 241-288.

5. Grothendieck, A., Topological Vector Spaces, Gordon and Breach, New York 1973.

6. Gupta, C., Convolution Operators and Holomorphic Mappings on a Banach Space, Séminaire d'Analyse Moderne 2, Univ. de Sherbrooke, Sherbrooke 1969.

7. Malgrange, B., Existence et approximation des solutions des équations aux derivées partielles et des équations de convolution, Ann. Inst. Fourier (Grenoble) 6 (1955-56), 271-355.

8. Martineau, A., Équations différentielles d'ordre infini, Bull. Soc. Math. France 95 (1967), 109-154.

9. Matos, M. C., On the Fourier-Borel Transformation and Spaces of Entire Functions in a Normed Space, pp. 139-170 in: G. I. Zapata (ed.), Functional Analysis, Holomorphy and Approximation Theory II, Proc. Rio de Janeiro 1981, North-Holland Math. Studies 86, North-Holland, Amsterdam 1984.

10. Matos, M. C., On convolution operators in spaces of entire functions of a given type and order, pp. 129-171 in: J. Mujica (ed.), Complex Analysis, Functional Analysis and Approximation Theory, Proc. Campinas 1984, North-Holland Math. Studies 125, North-Holland, Amsterdam 1986.

11. Matos, M. C., Absolutely Summing Mappings, Nuclear Mappings and Convolution Equations, Relatório de pesquisa 03/07, IMECC-UNICAMP, 2007. This book can be found at http://www.ime.unicamp.br/ matos. 
12. Nachbin, L., Lectures on the Theory of Distributions, Univ. de Recife, Recife 1964.

13. Nachbin, L., Topology on Spaces of Holomorphic Mappings, Ergebnisse Math. Grenzgebiete 47, Springer, New York 1969.

14. Robertson, A. P., and Robertson, W. J., Topological Vector Spaces, Cambridge Tracts Math. and Math. Physics 53, Cambridge University Press, New York 1973.

15. Mujica, X., Aplicações $\tau(p ; q)$-somantes e $\sigma(p)$-nucleares, Tese de Doutorado, Univ. Estadual de Campinas, 2006.

FACULDADE DE MATEMÁTICA

UNIVERSIDADE FEDERAL DE UBERLÂNDIA

38.400-902 - UBERLÂNDIA

BRAZIL

E-mail:vvfavaro@gmail.com

marques@famat.ufu.br 